\title{
Solar neutrino spectrum, sterile neutrinos and additional radiation in the Universe
}

\author{
P. C. de Holanda ${ }^{1}$ and A. Yu. Smirnov ${ }^{2}$ \\ (1) Instituto de Física Gleb Wataghin - UNICAMP, 13083-970 Campinas SP, Brazil \\ (2) The Abdus Salam International Centre for Theoretical Physics, I-34100 Trieste, Italy
}

\begin{abstract}
Recent results from the SNO, Super-Kamiokande and Borexino experiments do not show the expected upturn of the energy spectrum of events (the ratio $R \equiv$ $N_{\text {obs }} / N_{S S M}$ ) at low energies. At the same time, cosmological observations testify for possible existence of additional relativistic degrees of freedom in the early Universe: $\Delta N_{e f f}=1-2$. These facts strengthen the case of very light sterile neutrino, $\nu_{s}$, with $\Delta m_{01}^{2} \sim(0.7-2) \cdot 10^{-5} \mathrm{eV}^{2}$, which mixes weakly with the active neutrinos. The $\nu_{s}$ mixing in the mass eigenstate $\nu_{1}$ characterized by $\sin ^{2} 2 \alpha \sim 10^{-3}$ can explain an absence of the upturn. The mixing of $\nu_{s}$ in the eigenstate $\nu_{3}$ with $\sin ^{2} \beta \sim 0.1$ leads to production of $\nu_{s}$ via oscillations in the Universe and to additional contribution $\Delta N_{\text {eff }} \approx 0.7-1$ before the big bang nucleosynthesis and later. Such a mixing can be tested in forthcoming experiments with the atmospheric neutrinos as well as in future accelerator long baseline experiments. It has substantial impact on conversion of the supernova neutrinos.
\end{abstract}




\section{Introduction}

The large mixing angle (LMA) MSW solution [1, 2] has been established as the solution of the solar neutrino problem [3], [4], [5], [6], [7], [8], [9], [10]. In assumption of the CPT conservation KamLAND confirms this result [11, [12]. One of the main goals of further precision measurements of the solar neutrino fluxes is to search for possible deviations from the LMA predictions which would indicate new physics beyond the Standard Model with three mixed neutrinos. In particular, new physics can show up at the neutrino energies $E=(1-7) \mathrm{MeV}$, i.e. in the transition region between the matter dominated conversion and vacuum oscillations. Here direct measurements of the spectrum are absent or inprecise and possible deviations from the LMA predictions can be relatively large.

Some time ago in attempt to explain the low (about $2 \sigma$ ) rate in the Homestake experiment [3] in comparison to the LMA expectation as well as the absence of clear low energy upturn of the spectra of events at SuperKamiokande and SNO we have proposed a scenario with light sterile neutrino, $\nu_{s}$, which mixes weakly with active neutrinos [13. Conversion of $\nu_{e}$ to $\nu_{s}$ driven by the mass squared difference $\Delta m_{01}^{2} \sim(0.2-2) \cdot 10^{-5} \mathrm{eV}^{2}$ and mixing in the mass state $\nu_{1}, \sin ^{2} 2 \alpha \sim 10^{-3}$, leads to appearance of a dip in the $\nu_{e}-\nu_{e}$ survival probability in the range $(0.5-7) \mathrm{MeV}$ which explains the data.

After publication [13] several new experimental results have appeared which further support our proposal:

- Measurements of the solar neutrino spectrum by SuperKamiokande-III [14] with lower threshold still do not show the upturn.

- The SNO LETA analysis [15] gives even turn down of the spectrum in the two lowest energy bins.

- The Borexino measurements of the boron neutrino spectrum also hint some tendency of the spectral turn down [16].

Although separately these results are not statistically significant, being combined they can be considered an evidence of some new sub-leading effect.

At the same time, the cosmological observations indicate possible presence of additional radiation in the Universe in the epoch of last photon scattering. This is quantified by the effective number of neutrino species, $N_{\text {eff }}$, which is bigger than 3 . Combined analysis of WMAP-7, measurements of BAO (Baryon Acoustic Oscillations) and new value of the Hubble constant $H_{0}$ ) gives $N_{\text {eff }}=4.34_{-0.88}^{+0.86}$ [17]. WMAP-7 and Atacama Cosmology Telescope data lead to $N_{\text {eff }}=5.3 \pm 1.3$ (68\% C.L.) [18]. In the independent analysis [19] of these data the number of very light sterile neutrinos $\Delta N_{\text {eff }}=(0.02-2.2)(68 \%$ C.L.) has been obtained. All this confirms the earlier finding based on the WMAP-3 data: $N_{\text {eff }}=5.3_{-0.6-1.7-2.5}^{+0.4+2.1+3.8}[20]$. 
These results do not contradict the recent Big Bang Nucleosynthesis (BBN) bounds $N_{\text {eff }}=3.68_{-0.70}^{+0.80}[21$ (see discussion in [22] and theoretical considerations in [23]). Hence an additional radiation can be produced before the BBN epoch.

In this connection we revisit our proposal of very light sterile neutrinos. We show that mixing of this neutrino in mass states $\nu_{1}$ or/and $\nu_{2}$ can consistently improve description of the solar spectral data. We introduce mixing of this neutrino in the mass eigenstate $\nu_{3}$ which allows $\nu_{s}$ to be produced in the Early Universe with nearly equilibrium concentration, so that $\Delta N_{\text {eff }} \approx 1$.

The paper is organized as follows. In sect. 2 we consider properties of the $\nu_{e}$ conversion in the presence of $\nu_{s}$-mixing in the Sun generalizing our analysis in [13]. New feature, wiggles" in the survival probability, is described which appear for relatively large $\Delta m_{10}^{2}$ at the $E>5 \mathrm{MeV}$. In sect. 3 we obtain bounds on the $\nu_{s}$ parameters from the Borexino measurements of the $B e$-neutrino flux. Spectra of the solar neutrino events have been computed for different experiments and confronted with the data. In sect. 4. the mixing of $\nu_{s}$ in $\nu_{3}$ is introduced and phenomenological consequences of this mixing are studied, in particular, generation of $\nu_{s}$ in the Early Universe. The conclusion is given in sect. 5. In appendix we give some details of appearance of the wiggles in the survival probability.

\section{Sterile neutrino and conversion probabilities}

\section{$2.1 \quad$ Generalities}

Let us consider the system of 4 neutrinos $\nu_{f}=\left(\nu_{s}, \nu_{e}, \nu_{\mu}, \nu_{\tau}\right)$ mixed in the mass eigenstates $\nu_{i}, i=0,1,2,3$. The sterile neutrino, $\nu_{s}$, is mainly present in the mass eigenstate $\nu_{0}$ with mass $m_{0}$. It mixes weakly with active neutrinos and this mixing can be treated as small perturbation of the standard LMA structure.

Coherence of all mass eigenstates is lost on the way to the Earth. Therefore the $\nu_{e}^{-}$ survival probability at the surface of the Earth can be written as

$$
P_{e e}=\sum_{i}\left|A_{e i}^{S}\right|^{2}\left|U_{e i}\right|^{2}
$$

where $A_{e i}^{S}$ is the amplitude of the $\nu_{e} \rightarrow \nu_{i}$ transition inside the Sun and $U_{e i} \equiv\left\langle\nu_{e} \mid \nu_{i}\right\rangle$ is the element of the mixing matrix in vacuum. The quantities in eq. (1) satisfy the normalization conditions: $\sum_{i}\left|U_{e i}\right|^{2}=1$ and

$$
\sum_{i}\left|A_{e i}^{S}\right|=1
$$

During nights the solar neutrinos oscillate in the matter of the Earth. In this case $U_{e i}$ in eq. (11) should be substituted by the $\nu_{i} \rightarrow \nu_{e}$ oscillation probabilities inside the Earth, $U_{i e} \rightarrow A_{i e}^{E}$, so that

$$
P_{e e}=\sum_{i}\left|A_{e i}^{S}\right|^{2}\left|A_{i e}^{E}\right|^{2}
$$


In the production point the electron neutrino state can be represented in terms of the eigenstates in matter, $\nu_{i m}$, as

$$
\nu_{e}=\sum_{i} U_{e i}^{m} \nu_{i m}, \quad(i=0,1,2,3)
$$

where $U_{e i}^{m}$ is the mixing matrix element in matter in the production region. We denote by $\lambda_{i}$ the eigenvalues which correspond to the eigenstates $\nu_{i m}$. Introducing $A_{j i}$ - the amplitudes of $\nu_{i}^{m} \rightarrow \nu_{j}$ transitions inside the Sun we can write

$$
A_{e i}^{S}=\sum_{j} U_{e j}^{m} A_{j i}
$$

Insertion this expression into (2) gives

$$
P_{e e}=\sum_{i}\left|\sum_{j} U_{e j}^{m} A_{j i}\right|^{2}\left|A_{i e}^{E}\right|^{2} .
$$

In the adiabatic case $A_{i j}=\delta_{i j}$, so that

$$
P_{e e}=\sum_{i}\left|U_{e i}^{m}\right|^{2}\left|A_{i e}^{E}\right|^{2}
$$

For low energies we are interested in, the Earth matter effect is small and can be neglected in the first approximation.

In what follows we will introduce mixing of the sterile neutrino in different mass eigenstates. In computations of effects for solar neutrinos we neglect the 1-3 mixing and therefore consider the mixing of only three flavor states $\nu_{f}^{(3)}=\left(\nu_{s}, \nu_{e}, \nu_{a}\right)\left(\nu_{a}\right.$ is the mixture of $\nu_{\mu}$ and $\left.\nu_{\tau}\right)$. The mixing matrix which connects these states with the mass eigenstates, $\nu_{\text {mass }}=\left(\nu_{0}, \nu_{1}, \nu_{2}\right)$, can be parametrized as

$$
U^{(3)}=U_{\theta} U_{\alpha},
$$

where $U_{\alpha}$ is the matrix which mixes $\nu_{s}$ in $\nu_{1}$ or/and $\nu_{2}$ and $U_{\theta} \equiv U_{12}\left(\theta_{12}\right)$ is the standard LMA mixing (the rotation by the angle $\theta_{12}$ in the $\nu_{1}-\nu_{2}$ plane). The Hamiltonian of the system in the $\nu_{f}^{(3)}$ basis can be written as

$$
H_{f}=U_{\theta}^{\dagger} U_{\alpha}^{\dagger} H^{\operatorname{diag}} U_{\alpha} U_{\theta}+V
$$

where

$$
\begin{aligned}
H^{\text {diag }} & \equiv \operatorname{diag}\left(H_{0}, H_{1}, H_{2}\right)=\frac{1}{2 E} \operatorname{diag}\left(m_{0}^{2}, m_{1}^{2}, m_{2}^{2}\right) \\
V & \equiv \operatorname{diag}\left(0, V_{e}, V_{a}\right)
\end{aligned}
$$

are the diagonal matrices of the eigenvalues of the Hamiltonian in vacuum and the matter potentials correspondingly; $V_{e}=\sqrt{2} G_{F}\left(n_{e}-0.5 n_{n}\right)$ and $V_{a}=-(1 / \sqrt{2}) G_{F} n_{n}$, with $n_{e}$ and $n_{n}$ being the electron and neutron number densities. 


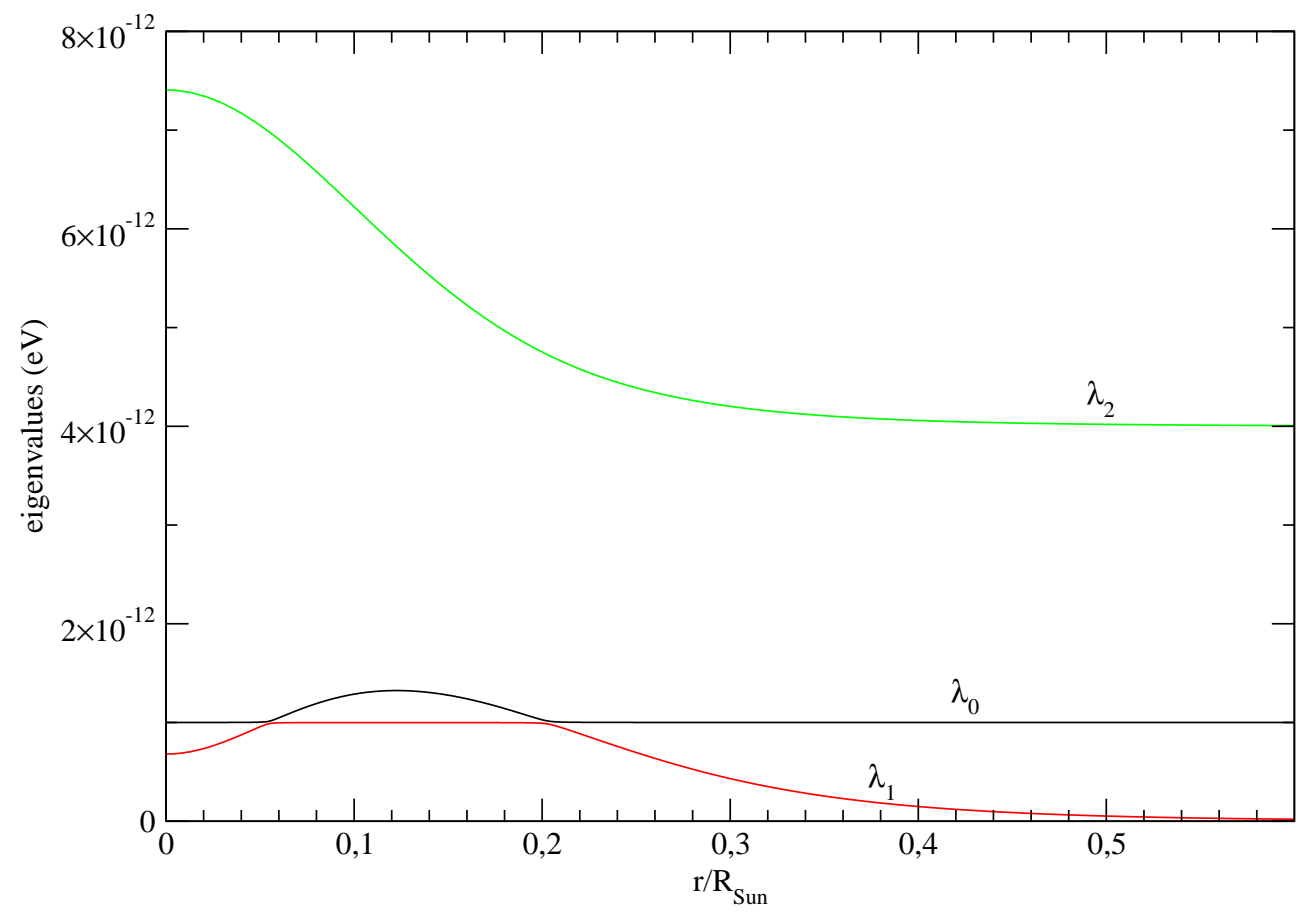

Figure 1: The level crossing scheme for the neutrino energy $E=10 \mathrm{MeV}$. Dependence of the eigenvalues of total Hamiltonian in matter, $\lambda_{i}$, on distance from the center of the Sun. The LMA neutrino oscillation parameters are taken as $\Delta m_{21}^{2}=8 \times 10^{-5} \mathrm{eV}^{2}$ and $\tan ^{2} \theta_{12}=0.44$, while sterile neutrino parameters are $R_{\Delta}=0.25$ and $\sin ^{2} 2 \alpha=10^{-3}$.

It is convenient to consider effects of sterile neutrino mixing in the basis rotated by the 1-2 mixing in matter $U_{\theta_{m}}:\left(\nu_{s}, \nu_{1 m}^{L M A}, \nu_{2 m}^{L M A}\right)$, which would diagonalize the Hamiltonian in the absence of mixing with sterile neutrinos (i.e. when $U_{\alpha}=I$ ). In this basis the Hamiltonian becomes

$$
H_{\alpha}=U_{\theta_{m}} H_{f} U_{\theta_{m}}^{\dagger}=U_{\theta_{m}} U_{\theta}^{\dagger} U_{\alpha}^{\dagger} H^{\text {diag }} U_{\alpha} U_{\theta} U_{\theta_{m}}^{\dagger}+U_{\theta_{m}} V U_{\theta_{m}}^{\dagger} .
$$

Since $U_{\alpha}$ is small rotation we can represent it as

$$
U_{\alpha}=I+U_{\delta}
$$

where $U_{\delta} \equiv U_{\alpha}-I$. Inserting this expression into (91) and taking the lowest order terms in $U_{\delta}$ we obtain

$$
H_{\alpha}=H_{m}^{d i a g}+H_{\delta}
$$

where

$$
H_{m}^{\operatorname{diag}}=\operatorname{diag}\left(H_{0}, \lambda_{1}^{L M A}, \lambda_{2}^{L M A}\right)
$$


is the Hamiltonian in the absence of mixing with sterile neutrino and

$$
H_{\delta} \equiv U_{\Delta \theta}^{\dagger} U_{\delta}^{\dagger} H^{\operatorname{diag}} U_{\Delta \theta}+\text { h.c. }
$$

is the correction to the Hamiltonian due to mixing with sterile neutrino. Here $\Delta \theta \equiv\left(\theta-\theta_{m}\right)$ and

$$
U_{\Delta \theta} \equiv U_{\theta} U_{\theta_{m}}^{\dagger}=\left(\begin{array}{ccc}
1 & 0 & 0 \\
0 & \cos \left(\theta-\theta_{m}\right) & -\sin \left(\theta-\theta_{m}\right) \\
0 & \sin \left(\theta-\theta_{m}\right) & \cos \left(\theta-\theta_{m}\right)
\end{array}\right)
$$

We denote the ratio of mass squared differences as

$$
R_{\Delta} \equiv \frac{\Delta m_{01}^{2}}{\Delta m_{21}^{2}}
$$

Depending on mass and mixing of the sterile neutrino (i.e. the form of $U_{\alpha}$ ) one can obtain several phenomenologically different possibilities.

\subsection{The case $m_{1}<m_{0}<m_{2}$}

This case corresponds to $R_{\Delta} \ll 1$ (as in [13]). For the neutrino with energy $E=10 \mathrm{MeV}$ the level crossing scheme which gives dependence of $\lambda_{i},(i=0,1,2)$ on the distance inside the Sun (or on the density), is shown in fig. 1. Details of construction of this scheme can be found in [13]. For other energies the scheme can be obtained from the one in Fig. 1 by shifting the picture with respect to the frame to the right with increase of energy and to the left with decrease of energy. According to fig. 1 for $E=10 \mathrm{MeV}$ the sterile neutrino level, $\lambda_{s}$, has two resonances - two crossings with the original (without $\nu_{s}$ ) level $\lambda_{1}^{L M A}$ : at smaller density $n_{l}^{R}$, and at higher density, $n_{h}^{R},\left(n_{h}^{R}>n_{l}^{R}\right)$. With increase of $\Delta m_{01}^{2}$ the density $n_{l}^{R}$ increases, whereas $n_{h}^{R}$ - decreases, they approach each other and then merge.

The flavor content of the eigenstates in matter, $\nu_{i m}$, i.e., the mixing matrix elements $U_{i \alpha}^{m}(n(r))$ as functions of density (distance from the center of the Sun) is shown in fig 2 , Notice that since the $\nu_{s}$ mixing in the $\nu_{2}$ is absent, the change of flavor of $\nu_{2 m}$ with density is the same as in the LMA case: $U_{2 \alpha}^{m}=U_{2 \alpha}^{m L M A}$. The intersections of the lines correspond to resonances.

Consider evolution of this system from the production point in the central region of the Sun to the solar surface. The eigenstates $\nu_{2 m}$ and $\nu_{3 m}$ evolve adiabatically

$$
\nu_{2 m} \rightarrow \nu_{2}, \quad \nu_{3 m} \rightarrow \nu_{3},
$$

i.e., $A_{2 j}=\delta_{2 j}, A_{3 j}=\delta_{3 j}$ and therefore according to (3)

$$
A_{e 2}^{S}=U_{e 2}^{m}, \quad A_{e 3}^{S}=U_{e 3}^{m} \approx U_{e 3} .
$$




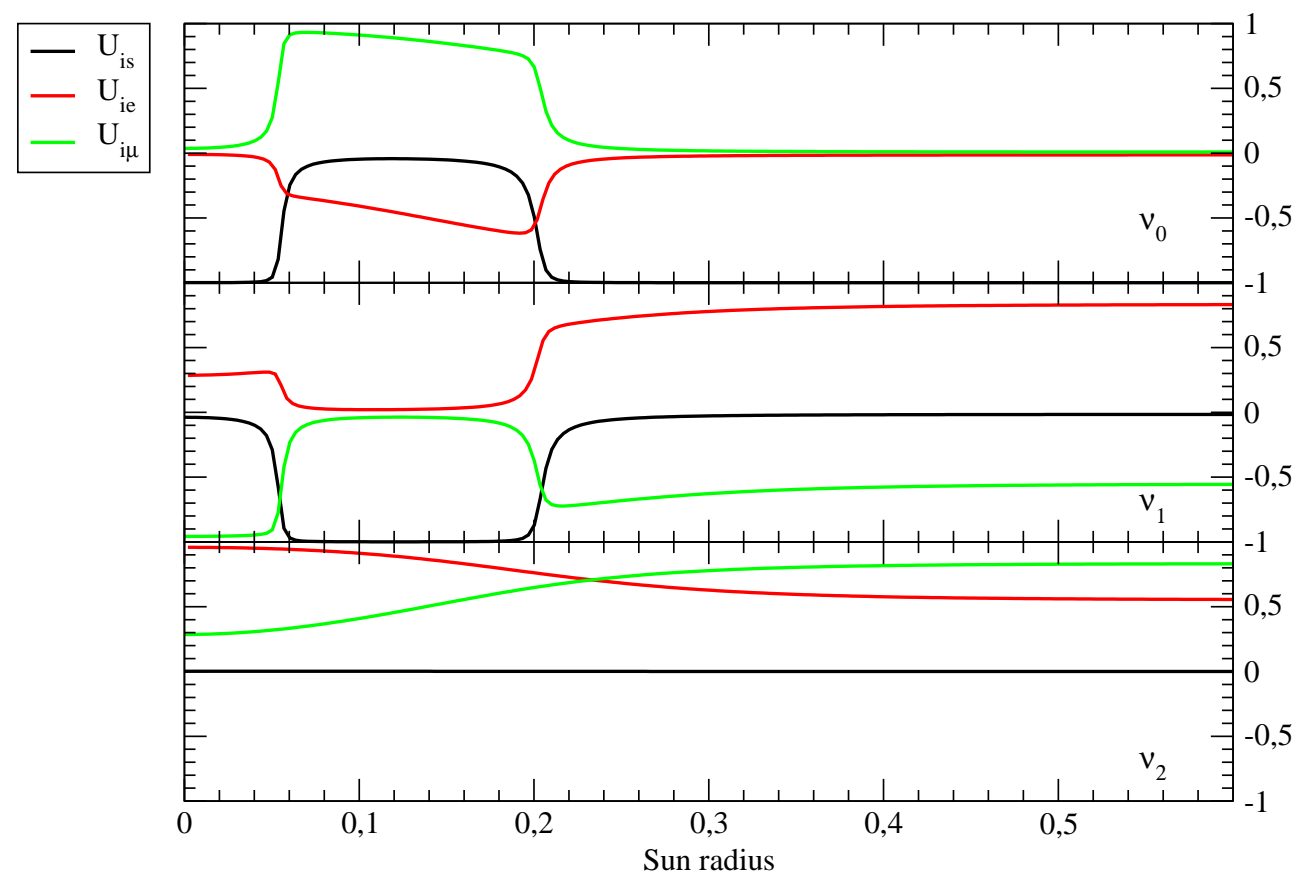

Figure 2: Flavor content of the mass eigenstates in matter for the same neutrino parameters as in fig. 1 .

In the last equality we have taken into account that due to large value of $\Delta m_{31}^{2}$ the 1-3 mixing is practically unaffected by the solar matter in the energy range of interest. Inserting the amplitudes (15) into (1) we obtain

$$
P_{e e}=\left|A_{e 1}^{S}\right|^{2}\left|U_{e 1}\right|^{2}+\left|A_{e 0}^{S}\right|^{2}\left|U_{e 0}\right|^{2}+\left|U_{e 2}^{m}\right|^{2}\left|U_{e 2}\right|^{2}+\left|U_{e 3}\right|^{4} .
$$

In turn, the amplitudes of $\nu_{e}$-transitions to $\nu_{1}$ and to $\nu_{0}$ can be written according to (3) as

$$
A_{e 1}^{S}=U_{e 1}^{m} A_{11}+U_{e 0}^{m} A_{01}, \quad A_{e 0}^{S}=U_{e 1}^{m} A_{10}+U_{e 0}^{m} A_{00} .
$$

Finally, insertion of (17) into (16) gives

$$
P_{e e}=\left|U_{e 1}^{m} A_{11}+U_{e 0}^{m} A_{01}\right|^{2}\left|U_{e 1}\right|^{2}+\left|U_{e 1}^{m} A_{10}+U_{e 0}^{m} A_{00}\right|^{2}\left|U_{e 0}\right|^{2}+\left|U_{e 2}^{m}\right|^{2}\left|U_{e 2}\right|^{2}+\left|U_{e 3}\right|^{4} .
$$

Since $\left|U_{e 0}\right|^{2} \sim 10^{-3}$ and $\left|U_{e 3}\right|^{4} \sim 4 \cdot 10^{-4}$, the probability (18) can be written approximately as

$$
P_{e e} \approx\left|U_{e 1}^{m} A_{11}+U_{e 0}^{m} A_{01}\right|^{2}\left|U_{e 1}\right|^{2}+\left|U_{e 2}^{m}\right|^{2}\left|U_{e 2}\right|^{2}
$$

With decrease of neutrino energy effectively the pattern in fig. 1 shifts to the left. Therefore at low energies there is no sterile neutrino resonances, the evolution proceeds 
adiabatically and

$$
P_{e e} \approx\left|U_{e 1}^{m}\right|^{2}\left|U_{e 1}\right|^{2}+\left|U_{e 2}^{m}\right|^{2}\left|U_{e 2}\right|^{2} .
$$

Furthermore, $U_{e 1}^{m} \approx U_{e 1}^{m L M A}$ and $U_{e 2}^{m} \approx U_{e 2}^{m L M A}$, where $U_{e i}^{m L M A}$ are mixing parameters in the pure LMA $(2 \nu)$ case without sterile neutrinos. So, $P_{e e} \approx P_{e e}^{L M A}$.

With increase of energy the low density resonance becomes effective (the corresponding level crossing scheme for $E=8 \mathrm{MeV}$ is shown in fig. 1 of our paper [13]). In the adiabatic case (relatively large $U_{01}$ ) we have $A_{01} \approx 0, A_{11} \approx 1$ and the expression for $P_{e e}$ in eq. (19) is reduced to the one in (20). However now $U_{e 1}^{m} \approx 0$ if neutrino is produced above the resonance layer, and consequently, $P_{e e} \approx\left|U_{e 2}^{m}\right|^{2}\left|U_{e 2}\right|^{2}$.

In the non-adiabatic case:

$$
P_{e e} \approx\left|U_{e 0}^{m} A_{01}\right|^{2}\left|U_{e 1}\right|^{2}+\left|U_{e 2}^{m}\right|^{2}\left|U_{e 2}\right|^{2} \approx\left|U_{e 1}^{m L M A} A_{01}\right|^{2}\left|U_{e 1}\right|^{2}+\left|U_{e 2}^{m}\right|^{2}\left|U_{e 2}\right|^{2},
$$

where we have taken into account that $U_{e 0}^{m} \approx U_{e 1}^{m L M A}$. In the case of strong adiabaticity violation (for very small $U_{01}$ ) when $A_{01} \approx 1$, the probability in (21) is reduced to the standard LMA probability.

With further increase of energy at $E \gtrsim 9 \mathrm{MeV}$ two sterile resonances are realized and the amplitudes $A_{01}$ and $A_{11}$ can be written in terms of transition amplitudes in each resonance $A_{i j}^{(a)}(a=1,2)$ as

$$
A_{11}=A_{11}^{(2)} A_{11}^{(1)}+A_{10}^{(2)} A_{01}^{(1)}, \quad A_{01}=A_{00}^{(2)} A_{01}^{(1)}+A_{01}^{(2)} A_{11}^{(1)} .
$$

One can get different results depending on the adiabaticity conditions in each resonance. If the crossings are adiabatic, $A_{01} \approx 0, A_{11} \approx 1$, we obtain from (19)

$$
P_{e e} \approx\left|U_{e 1}^{m}\right|^{2}\left|U_{e 1}\right|^{2}+\left|U_{e 2}^{m}\right|^{2}\left|U_{e 2}\right|^{2}
$$

This expression is similar to the one for the standard LMA case, however now $\left|U_{e 1}^{m}\right|^{2}=$ $1-\left|U_{e 2}^{m}\right|^{2}-\left|U_{e 0}^{m}\right|^{2}=\left|U_{e 1}^{m L M A}\right|^{2}-\left|U_{e 0}^{m}\right|^{2}$, and consequently,

$$
P_{e e}=P_{e e}^{L M A}-\left|U_{e 1}\right|^{2}\left|U_{e 0}^{m}\right|^{2} .
$$

Here the second term describes the dip in the adiabatic case. (Notice that due to smallness of $\nu_{s}$ mixing in vacuum $U_{e 1} \approx U_{e 1}^{L M A}$.)

If adiabaticity is strongly broken in both resonances due to smallness of sterile mixing, then $A_{10}^{(2)} \approx A_{01}^{(1)} \approx 1$ and $A_{11}^{(2)} \approx A_{00}^{(2)} \approx A_{11}^{(1)} \approx 0$. Therefore according to $(22), A_{01} \approx 0$, $A_{11} \approx 1$, and as in the adiabatic case:

$$
P_{e e} \approx\left|U_{e 1}^{m}\right|^{2}\left|U_{e 1}\right|^{2}+\left|U_{e 2}^{m}\right|^{2}\left|U_{e 2}\right|^{2}
$$

However, now the mixing parameters are approximately equal to the standard LMA parameters without sterile neutrinos. Therefore $P_{e e} \approx P_{e e}^{L M A}$. 
In the case of mixing of $\nu_{s}$ in $\nu_{1}$ and $\nu_{0}$ only, the matrix $U_{\alpha}$ equals

$$
U_{\alpha}=\left(\begin{array}{ccc}
\cos \alpha & \sin \alpha & 0 \\
-\sin \alpha & \cos \alpha & 0 \\
0 & 0 & 1
\end{array}\right)
$$

Explicitly the flavor mixing can be parameterized as

$$
\begin{aligned}
& \nu_{0}=\cos \alpha \nu_{s}+\sin \alpha\left(\cos \theta_{12} \nu_{e}-\sin \theta_{12} \nu_{a}\right) \\
& \nu_{1}=\cos \alpha\left(\cos \theta_{12} \nu_{e}-\sin \theta_{12} \nu_{a}\right)-\sin \alpha \nu_{s} \\
& \nu_{2}=\sin \theta_{12} \nu_{e}+\cos \theta_{12} \nu_{a} .
\end{aligned}
$$

Here $\nu_{e}$ and $\nu_{a}$ (a combination of $\nu_{\mu}$ and $\nu_{\tau}$ ) mix with the angle $\theta_{12}$ in the mass eigenstates $\nu_{1}$ and $\nu_{2}$ having the mass split $\Delta m_{12}^{2}$. In terms of these mixing angles:

$$
U_{e 1}=\cos \alpha \cos \theta_{12}, \quad U_{e 0}=\sin \alpha \cos \theta_{12}, \quad U_{e 2}=\sin \theta_{12} .
$$

With this parametrization eqs. (19) and (23) reproduce the corresponding results of our previous paper [13].

From eqs. (11- 14) we find using (24) that the Hamiltonian in the rotated basis equals

$$
H_{\alpha}=\left(\begin{array}{ccc}
0 & -\frac{\Delta m_{01}^{2}}{2 E} \sin \alpha \cos \left(\theta-\theta_{m}\right) & -\frac{\Delta m_{01}^{2}}{2 E} \sin \alpha \sin \left(\theta-\theta_{m}\right) \\
\ldots & \lambda_{1}^{L M A}-H_{0} & 0 \\
\ldots & \ldots & \lambda_{2}^{L M A}-H_{0}
\end{array}\right) .
$$

Due to smallness of $\alpha$ the off-diagonal terms are much smaller than the diagonal ones. If $R_{\Delta} \ll 1$, so that $H_{0}$ is close to $\lambda_{1}^{L M A}$ and crosses this level, there is no crossing of $\lambda_{2}^{L M A}$ and $H_{0}$ levels, and the state $\nu_{2 m}^{L M A}$ decouples. Then mixing of $\nu_{s}$ and $\nu_{1 m}^{L M A}$ is determined by

$$
\sin \alpha \frac{\Delta m_{01}^{2}}{2 E} \cos \left(\theta-\theta_{m}\right)=\sin \alpha \frac{\Delta m_{21}^{2}}{2 E} R_{\Delta} \cos \left(\theta-\theta_{m}\right)
$$

Since the transition occurs in the resonance region, $\theta_{m}$ should be taken at the density which corresponds to the sterile neutrino resonance. The expression for mixing (26) allows to understand behavior of the conversion probability on $R_{\Delta}, \alpha$ and neutrino energy.

In figs. 3, 4 we show results of numerical computations of the $\nu_{e}$ survival probability $P_{e e}$ as functions of the neutrino energy. We have performed complete integration of the evolution equations for the $3 \nu$-system and also made averaging over the production region of the Sun. The following analytical consideration allows to understand the numerical results shown in fig. 3. The $\nu_{s}$-mixing and $\nu_{e} \rightarrow \nu_{s}$ conversion lead to appearance of a dip in the energy dependence of the $\nu_{e}$-survival probability. The survival probability in the pure LMA case is given by

$$
P_{e e}^{L M A} \approx\left|U_{e 1}^{m L M A}\right|^{2}\left|U_{e 1}^{L M A}\right|^{2}+\left|U_{e 2}^{m L M A}\right|^{2}\left|U_{e 2}^{L M A}\right|^{2}+\left|U_{e 3}\right|^{4} .
$$




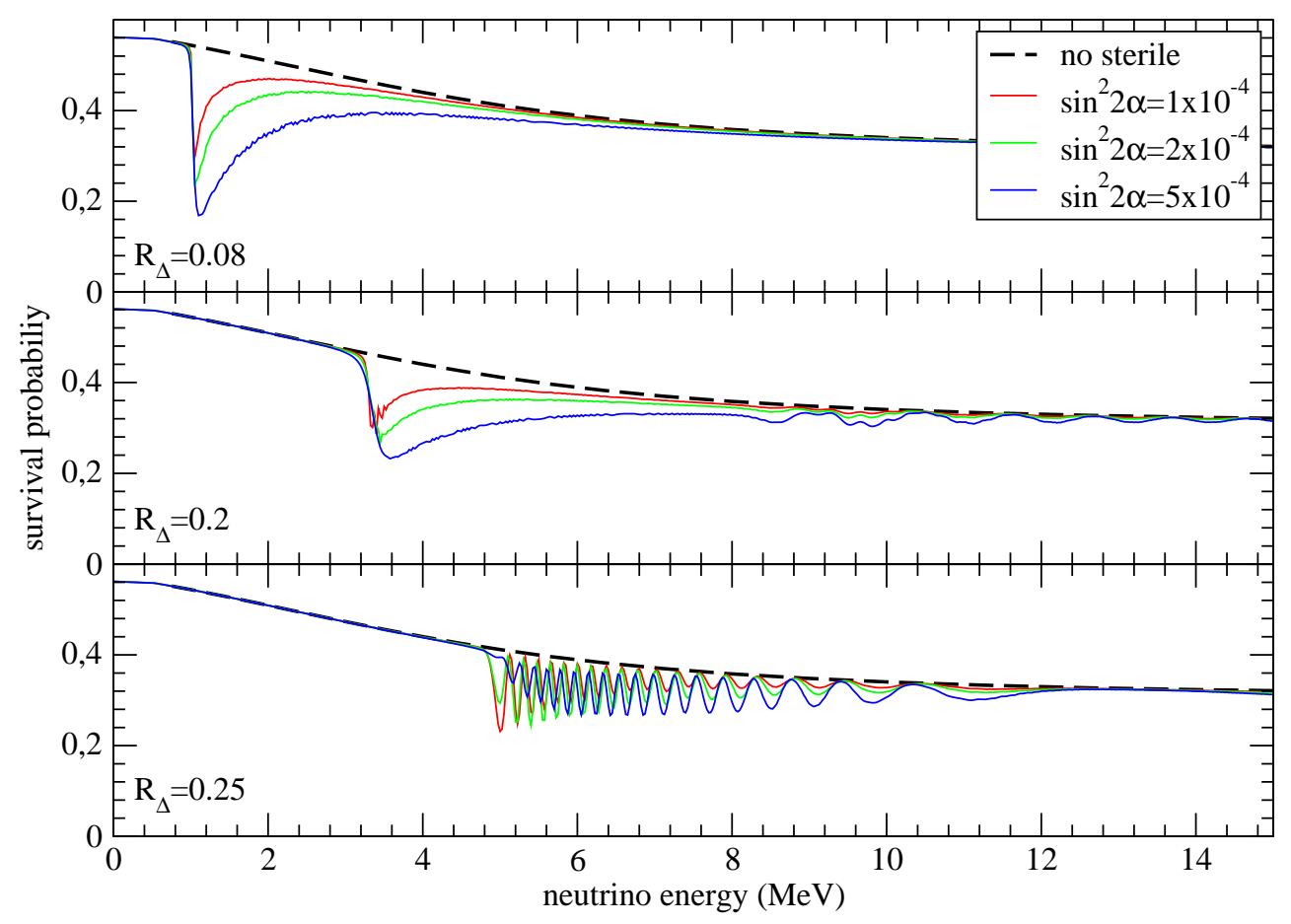

Figure 3: The survival probability of the electron neutrino as function of neutrino energy for different values of the sterile-active mixing parameter $\sin ^{2} 2 \alpha$ and mass scale $R_{\Delta} \ll 1$. The active neutrino parameters are $\Delta m_{21}^{2}=8 \times 10^{-5} \mathrm{eV}^{2}$ and $\tan ^{2} \theta=0.44$.

Since $\nu_{s}$ mixing in the $\nu_{2}$ is absent, $U_{e 2}^{L M A}=U_{e 2}$ and $U_{e 2}^{m L M A}=U_{e 2}^{m}$ and the probability (27) can be rewritten as

$$
P_{e e}^{L M A} \approx\left|U_{e 1}^{0 m}\right|^{2}\left|U_{e 1}^{0}\right|^{2}+\left|U_{e 2}^{m}\right|^{2}\left|U_{e 2}\right|^{2}+\left|U_{e 3}\right|^{4} .
$$

Using normalization conditions

$$
\left|A_{e 1}^{S}\right|^{2}+\left|A_{e 0}^{S}\right|^{2}+\left|U_{e 2}^{m}\right|^{2}=1
$$

(we neglect 1-3 mixing here) and $\sum_{i}\left|U_{e i}\right|^{2}=1(\mathrm{i}=0,1,2,3)$ as well as the expressions in eqs. (16) and (28) we can find difference of the probabilities with and without sterile neutrino effect:

$$
\begin{aligned}
\Delta P_{e e} & \equiv P_{e e}^{L M A}-P_{e e}=\left|A_{e 0}^{S}\right|^{2}\left(1-\left|U_{e 2}\right|^{2}\right)-\left|U_{e 0}\right|^{2}\left(2\left|A_{e 0}^{S}\right|^{2}-1-\left|U_{e 2}^{m}\right|^{2}\right) \\
& \approx\left|A_{e 0}^{S}\right|^{2}\left(1-\left|U_{e 2}\right|^{2}\right) \approx P_{e s}\left(1-\left|U_{e 2}\right|^{2}\right),
\end{aligned}
$$

where $P_{e s} \approx\left|A_{e 0}^{S}\right|^{2}$ is the probability of $\nu_{e} \rightarrow \nu_{s}$ transition. The quantity $\Delta P_{e e}$ describes the dip which has the following properties (see fig. 3 and also discussion in [13]): 


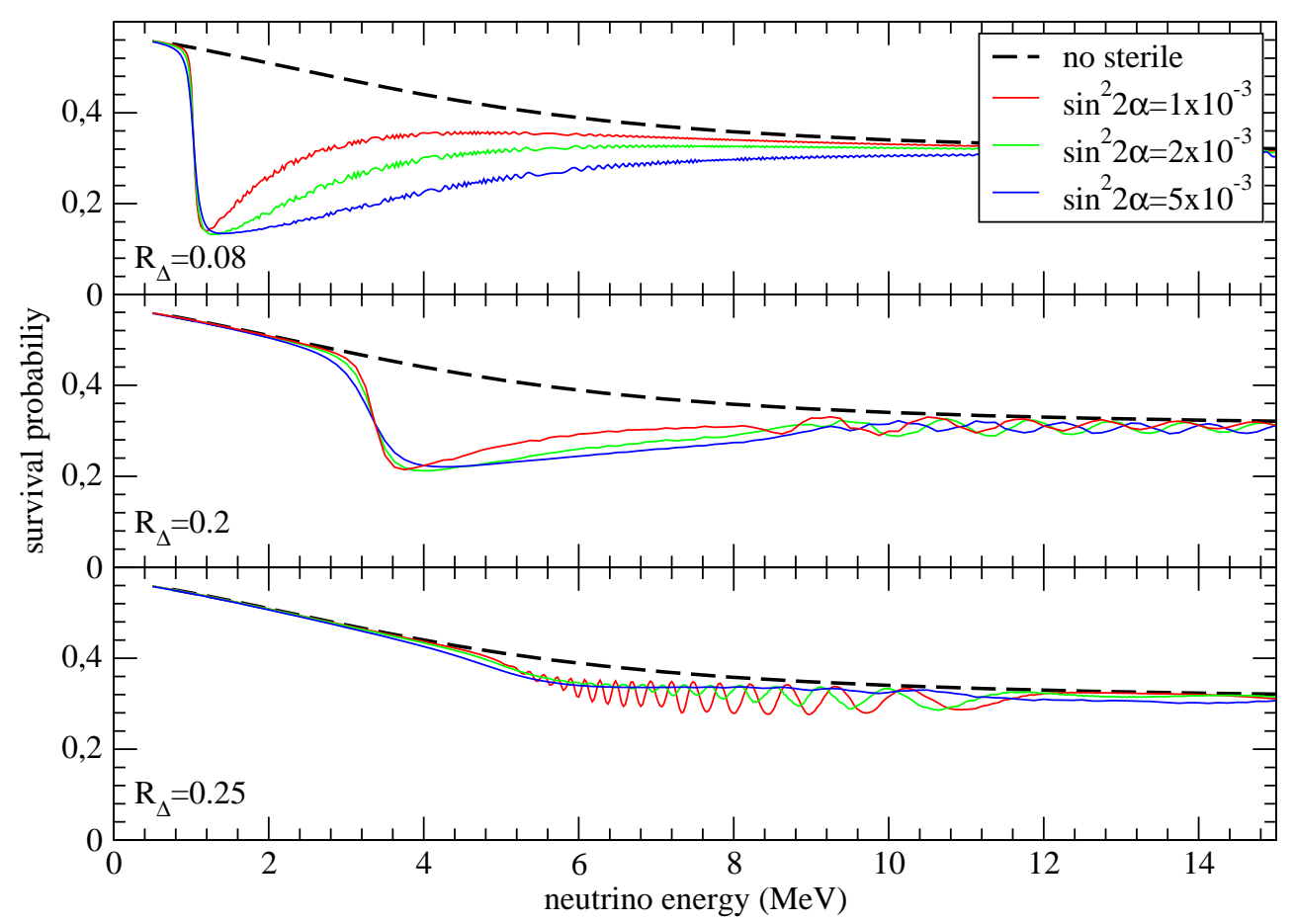

Figure 4: The same as in fig. 3 for higher values of mixing angle $\alpha$.

1. A position of the dip (its low energy edge) is given by the low density resonance taken at the central density of the Sun $E_{l}\left(n_{c}\right)$. With increase of $\Delta m_{01}^{2}$ the dip shifts to higher energies.

2. Maximal suppression in the dip depends on $R_{\Delta}$ and $\alpha$. For small $R_{\Delta}$ (large spit between the two resonances) and large $\alpha\left(\sin ^{2} 2 \alpha>10^{-3}\right)$ the absolute minimum can be achieved at the adiabatic crossing of the $\nu_{s}$-resonances. With increase of $R_{\Delta}$ (smaller split of the resonances) or/and decrease of $\alpha$ (stronger violation of the adiabaticity) a suppression in the dip weakens. Also with decrease of $\alpha$ the dip becomes narrower.

3. For large $\Delta m_{01}^{2}$ and relatively small $\alpha$ the survival probability as function of the neutrino energy has wiggles (see fig. 33). The wiggles are result of interference of the two amplitudes in the first term of (19) which develops over finite space interval. Indeed, according to (19) there are two channels of transition of $\nu_{e}$ to $\nu_{1}$ :

(i) $\nu_{e}$ has admixture $U_{e 1}^{m}$ in $\nu_{1 m}$, the latter adiabatically evolves to $\nu_{1}: \nu_{e} \rightarrow \nu_{1 m} \rightarrow \nu_{1}$, and the amplitude equals $U_{e 1}^{m} A_{11}$.

(ii) $\nu_{e}$ has admixture $U_{e 0}^{m}$ in $\nu_{0 m}$; this state transforms to $\nu_{1}$ due to non-adiabatic tran- 
sition: $\nu_{e} \rightarrow \nu_{0 m} \rightarrow \nu_{1}$. The corresponding amplitude is $U_{e 0}^{m} A_{01}$.

The two contributions to the amplitude interfere leading to the oscillatory dependence of the probability on energy (wiggles). Introducing $P_{01} \equiv\left|A_{01}\right|^{2}$, so that $\left|A_{11}\right|^{2}=1-P_{01}$, we can rewrite the probability (19) as

$$
P_{e e} \approx\left|U_{e 1}\right|^{2}\left[\left|U_{e 1}^{m}\right|^{2}\left(1-P_{01}\right)+\left|U_{e 0}^{m}\right|^{2} P_{01}+U_{e 1}^{m} U_{e 0}^{m} \cos \phi \sqrt{P_{01}\left(1-P_{01}\right)}\right]+\left|U_{e 2}\right|^{2}\left|U_{e 2}^{m}\right|^{2},
$$

where $\phi \equiv \arg \left(A_{01}^{*} A_{11}\right)$ and we assumed for simplicity that $U_{e 1}^{m}$ and $U_{e 0}^{m}$ are real. The oscillatory behavior follows from the energy dependence of the phase $\phi$. The key point is that the phase is collected over restricted space interval, $L$, and therefore is not averaged out even after integration over the production region. Indeed, the phase $\phi$ is acquired from the neutrino production point to the second (low density) resonance. Below the second resonance (in density) both "trajectories" (channels of transition) coincide. Appearance of the wiggles requires the adiabaticity violation. In the adiabatic case $A_{01}=0$ and only one channel exists. Unfortunately, it will be difficult, if possible, to observe experimentally these wiggles. Some more details concerning the wiggles are presented in the Appendix.

If $\nu_{s}$ mixes in $\nu_{2}$, then

$$
U_{\alpha}=\left(\begin{array}{ccc}
\cos \alpha^{\prime} & 0 & \sin \alpha^{\prime} \\
0 & 1 & 0 \\
-\sin \alpha^{\prime} & 0 & \cos \alpha^{\prime}
\end{array}\right)
$$

and the Hamiltonian can be obtained from (25) by substitutions

$$
\cos \left(\theta-\theta_{m}\right) \rightarrow \sin \left(\theta-\theta_{m}\right), \quad \sin \left(\theta-\theta_{m}\right) \rightarrow-\cos \left(\theta-\theta_{m}\right), \quad \Delta m_{01}^{2} \rightarrow \Delta m_{02}^{2} .
$$

Again the state $\nu_{2 m}$ decouples and $\nu_{s}-\nu_{1 m}^{L M A}$ mixing is given by

$$
\sin \alpha^{\prime} \frac{\Delta m_{02}^{2}}{2 E} \sin \left(\theta-\theta_{m}\right)=\sin \alpha^{\prime} \frac{\Delta m_{21}^{2}}{2 E}\left(1-R_{\Delta}\right) \sin \left(\theta-\theta_{m}\right) .
$$

Notice that this mixing appears due to matter effect and it is absent in vacuum when $\theta_{m} \rightarrow \theta$. It happens that for values of $R_{\Delta}$ we are considering $\left(1-R_{\Delta}\right) \sin \left(\theta-\theta_{m}\right) \approx R_{\Delta} \cos \left(\theta-\theta_{m}\right)$ and therefore the probabilities in this case are very similar to those shown in figs. 3, 4,

\section{$2.3 \quad m_{0}>m_{2}>m_{1}$ and other possibilities}

For $m_{0}>m_{2}>m_{1}$ the ratio $R_{\Delta}>1$. Since below the LMA resonance $\lambda_{2}=\lambda_{2}^{L M A}$ and $V_{n}$ have practically the same dependences on density (radius) (see fig. (1), there is only one crossing of $\lambda_{s}$ with $\lambda_{2}^{L M A}$, and there is no crossings for $\Delta m_{02}^{2}<0$. Now the evolution of states $\nu_{1 m}$ and $\nu_{3 m}$ is adiabatic, so that

$$
A_{e 1}^{S} \approx U_{e 1}^{m}=U_{e 1}^{0 m}, \quad A_{e 3}^{S} \approx U_{e 3} .
$$


Consequently,

$$
P_{e e}=\left|U_{e 1}^{m}\right|^{2}\left|U_{e 1}\right|^{2}+\left|A_{e 2}^{S}\right|^{2}\left|U_{e 2}\right|^{2}+\left|A_{e 0}^{S}\right|^{2}\left|U_{e 0}\right|^{2}+\left|U_{e 3}\right|^{4},
$$

where

$$
A_{e 2}^{S}=U_{e 2}^{m} A_{22}+U_{e 0}^{m} A_{02}, \quad A_{e 0}^{S}=U_{e 2}^{m} A_{20}+U_{e 0}^{m} A_{00} .
$$

These expressions are similar to the expressions in (16) and (3) with interchange of indexes $1 \leftrightarrow 2$.

In the adiabatic case we have

$$
P_{e e}=\left|U_{e 1}^{m}\right|^{2}\left|U_{e 1}\right|^{2}+\left|U_{e 2}^{m}\right|^{2}\left|U_{e 2}\right|^{2}+\left|U_{e 0}^{m}\right|^{2}\left|U_{e 0}\right|^{2}+\left|U_{e 3}\right|^{4} .
$$

Now the effect of sterile neutrino is due to difference of $U_{e 2}^{m}$ and $U_{e 2}^{m L M A}:\left|U_{e 2}^{m}\right|^{2}=\left|U_{e 2}^{m L M A}\right|^{2}-$ $\left|U_{e 0}^{m}\right|^{2}$. In the strongly non-adiabatic case $A_{20} \approx A_{02} \approx 1$ and

$$
P_{e e}=\left|U_{e 1}^{m}\right|^{2}\left|U_{e 1}\right|^{2}+\left|U_{e 2}^{m}\right|^{2}\left|U_{e 0}\right|^{2}+\left|U_{e 0}^{m}\right|^{2}\left|U_{e 2}\right|^{2}+\left|U_{e 3}\right|^{4},
$$

with $U_{e 2}^{m} \approx 0$, and $U_{e 0}^{m} \approx U_{e 2}^{m L M A}$, so that (32) is reduced to the LMA probability.

For $\nu_{s}$-mixing in $\nu_{0}$ and $\nu_{1}$ the Hamiltonian is given by the same expression as in eq. (25). However, now $H_{0}$ crosses $\lambda_{2}^{L M A}$ and the state $\nu_{1 m}^{L M A}$ decouples. According to (25), the mixing of $\nu_{s}$ and $\nu_{2 m}^{L M A}$ is determined by

$$
\sin \alpha \frac{\Delta m_{01}^{2}}{2 E} \sin \left(\theta-\theta_{m}\right)=\sin \alpha \frac{\Delta m_{21}^{2}}{2 E} R_{\Delta} \sin \left(\theta-\theta_{m}\right),
$$

and this mixing is due to matter effect. For $\nu_{s}-$ mixing in $\nu_{0}$ and $\nu_{2}$ performing substitutions (30) we obtain the $\nu_{s}-\nu_{2 m}^{L M A}$ mixing

$$
\sin \alpha^{\prime} \frac{\Delta m_{02}^{2}}{2 E} \cos \left(\theta-\theta_{m}\right)=\sin \alpha^{\prime} \frac{\Delta m_{21}^{2}}{2 E}\left(1-R_{\Delta}\right) \cos \left(\theta-\theta_{m}\right) .
$$

In the first case, eq. (33), the mixing $\propto R_{\Delta} \sin \left(\theta-\theta_{m}\right)$ is larger than in second case (34): $\propto\left(1-R_{\Delta}\right) \cos \left(\theta-\theta_{m}\right)$, since $R_{\Delta} \sim 1$. Furthermore, the first mixing increases with energy: the sterile resonance is above the LMA resonance and therefore $\theta_{m}>45^{\circ}$; this angle, and consequently $\left|\sin \left(\theta-\theta_{m}\right)\right|$, increase. As a result, the effect does not disappear at high energies (see 5 and 6 ).

In comparison to the case $R_{\Delta} \ll 1$, now $\nu_{e}$ has smaller admixture in $\nu_{2},\left|U_{e 2}\right|<\left|U_{e 1}\right|$, however the initial admixture of $\nu_{e}$ in $\nu_{2 m}$ can be larger: $\left|U_{e 2}^{m}\right|>\left|U_{e 1}^{m}\right|$. Therefore the overall effect is large (see figs 5 and (6). Indeed here we have only one level crossing and improvement of the adiabaticity in the resonance leads to stronger transition. With increase of $\Delta m_{02}^{2}$ and therefore, $R_{\Delta}$, the dip moves to high energies but the resonance shifts to higher densities, i.e., to the central regions of the Sun where the density gradient is smaller and adiabaticity is better. Here substantial change of the probability exists for smaller mixing angles. 


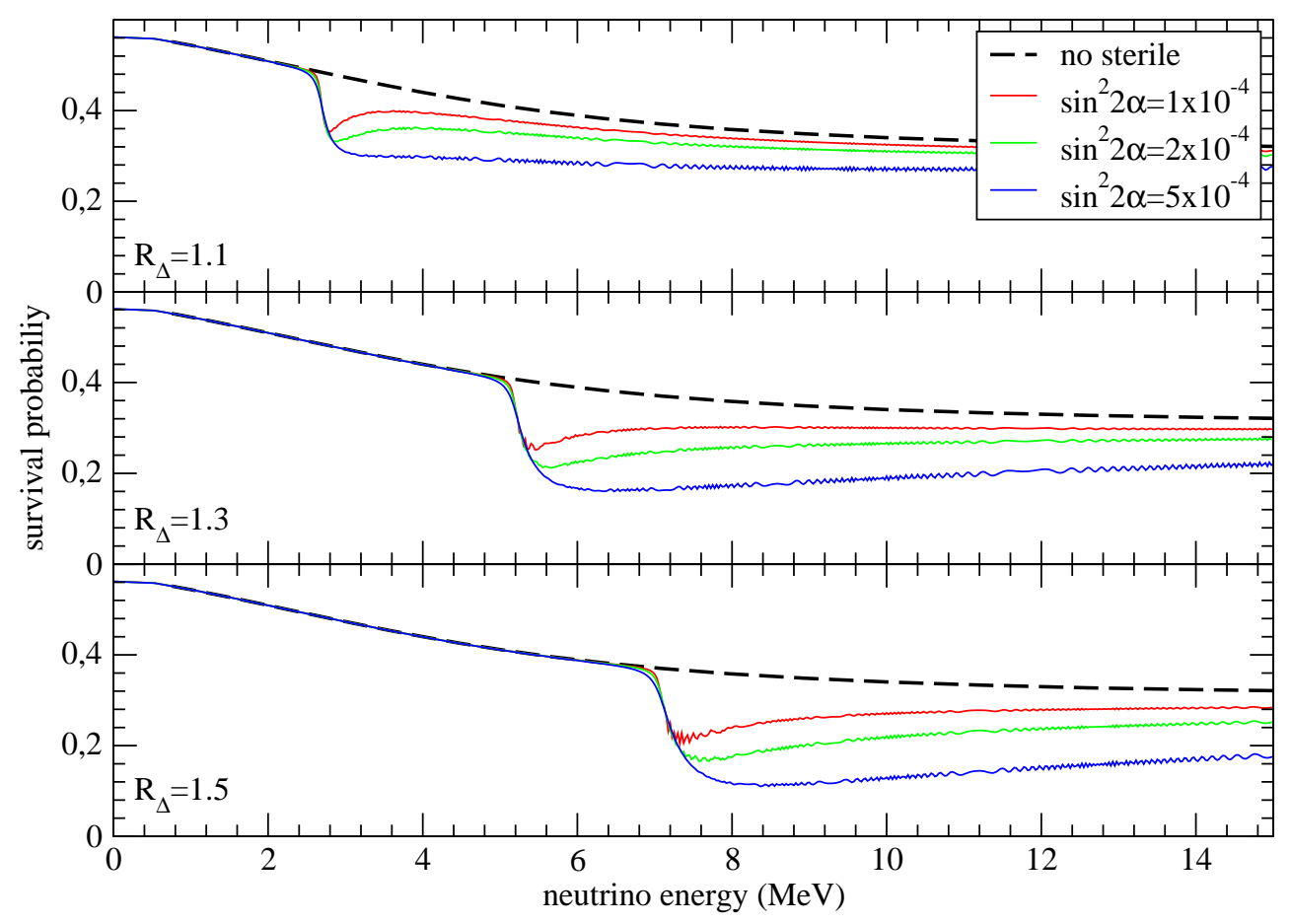

Figure 5: The same as in fig. 3 for $R_{\Delta}>1$.

If $m_{0}<m_{1}<m_{2}$, so that $\Delta m_{01}^{2}<0$, the sterile level $\lambda_{s}$ crosses $\nu_{1 m}^{L M A}$ at high densities only

$$
n_{h}^{R} \approx 2 n^{L M A}\left(1+R_{\Delta}\right)
$$

The resonance energy equals

$$
E \approx E_{R}^{L M A} \frac{2 n_{e}}{n_{n}}
$$

where $n_{n}$ is the number density of neutrons. In this case we have the same general expressions for the survival probability as in (18) and (19). Consequently, the expressions for $P_{e e}$ in adiabatic and non-adiabatic limits coincide with those in e.g. (21) for one sterile resonance. However the dip here is at high energies.

In the case of flavor mixing, that is the mixing of $\nu_{s}$ with $\nu_{e}, \nu_{a}$ the matrices $U_{\theta}$ and $U_{\alpha}$ should be permuted, so that $U^{(3)}=U_{\alpha} U_{\theta}$ (compare with (6a $)$ ). It can be shown that now the off-diagonal elements of the Hamiltonian $H_{\alpha}$ contain terms with $\Delta m_{01}^{2}$ and $\Delta m_{02}^{2}$ simultaneously. As a result, the probabilities have energy dependences which are intermediate between those for mixings in mass states $\nu_{1}$ and $\nu_{2}$. 


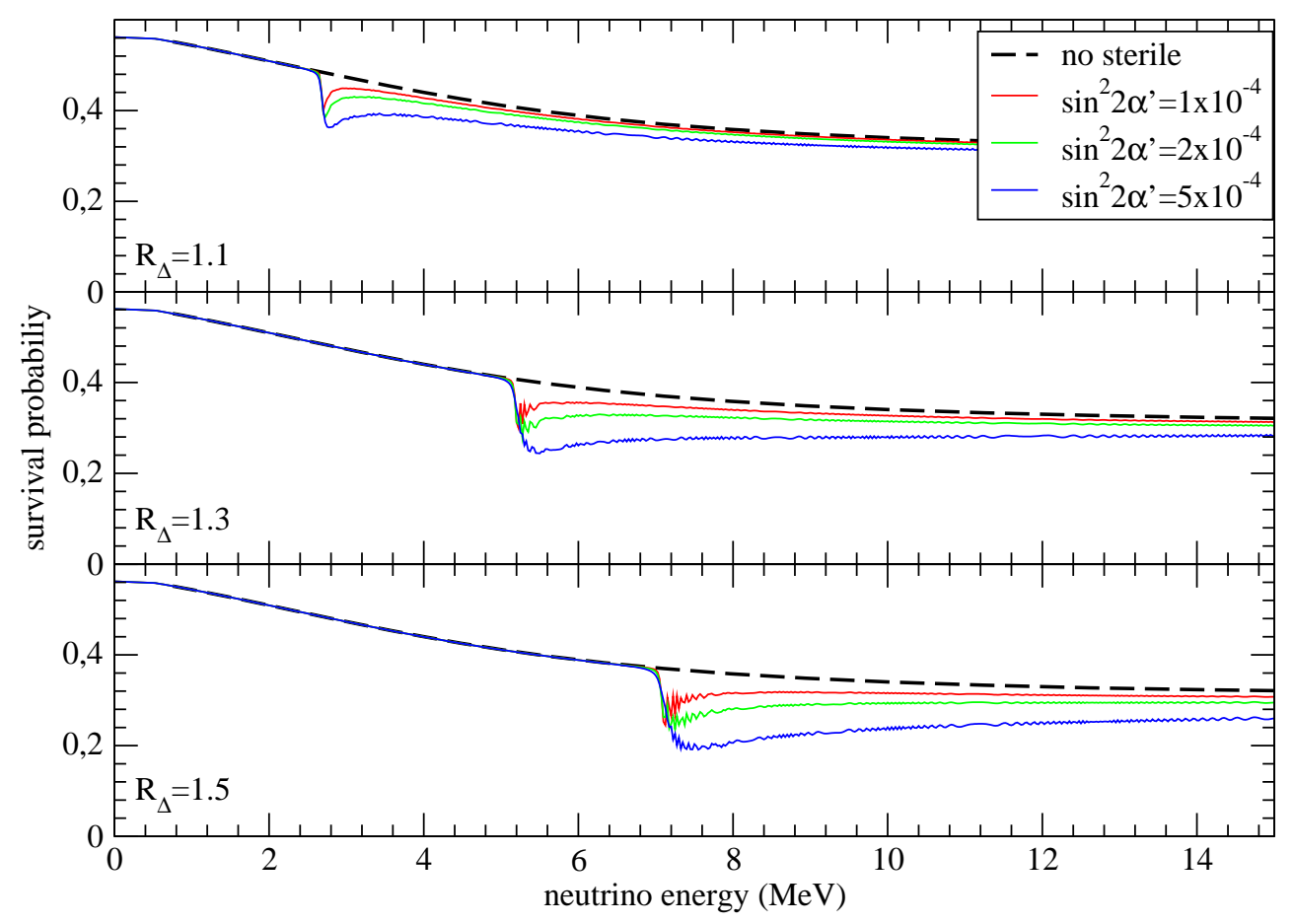

Figure 6: The same as in fig. 4 for $R_{\Delta}>1$ and $\nu_{s}$ mixing in $\nu_{2}$.

\section{Solar neutrino data and sterile neutrino effect}

In what follows we will consider scenario with $m_{1}<m_{0}<m_{2}$. This possibility gives better description of the data: it leads to significant modification of the survival probability in the transition region and weakly affects spectra at high energies.

\subsection{Borexino measurements of the Be-neutrino line}

The results of Borexino experiment [10] are in very good agreement with prediction based on the LMA solution and the Standard Solar Model. Within the error bars no additional suppression of the flux has been found on the top of $P_{e e}^{L M A}$. In Borexino (and other experiments based on the $\nu e$-scattering) the ratio of the numbers of events with and without conversion can be written as

$$
R_{\text {Borexino }}=P_{e e}(1-r)+r-r P_{e s}
$$


where $r \equiv \sigma\left(\nu_{\mu} e-\nu_{\mu} e\right) / \sigma\left(\nu_{e} e-\nu_{e} e\right)$ is the ratio of cross-sections. Using Eq. (35) we find an additional suppression of the Borexino rate in comparison with the pure LMA case [13]:

$$
\Delta R_{\text {Borexino }} \equiv R_{\text {Borexino }}^{L M A}-R_{\text {Borexino }}=(1-r) \Delta P_{e e}+r P_{e s} \approx \Delta P_{e e}\left(1+r \tan ^{2} \theta_{12}\right) .
$$

In fig. [7 we show dependence of the survival probability at $E=E_{B e}$ as function of $R_{\Delta}$ for two different values of the mixing angle $\alpha$. We show the Borexino bounds on $P_{e e}$ obtained from the experimental result [16] and relation (35). According to this figure for $\sin ^{2} 2 \alpha=10^{-3}$ the range $R_{\Delta}=0.005-0.072$ is excluded at $1 \sigma$ level. For $\sin ^{2} 2 \alpha=5 \cdot 10^{-3}$ we obtain slightly larger exclusion interval: $R_{\Delta}=0.001-0.075$. The $B e-$ neutrino line can not be in the dip or the dip should be shallow which then will have little impact on the higher energy spectrum. So, essentially the allowed values of masses (which influence the upturn) are

$$
R_{\Delta} \geq 0.075, \text { or } \Delta m_{01}^{2} \geq 0.5 \cdot 10^{-5} \mathrm{eV}^{2} .
$$

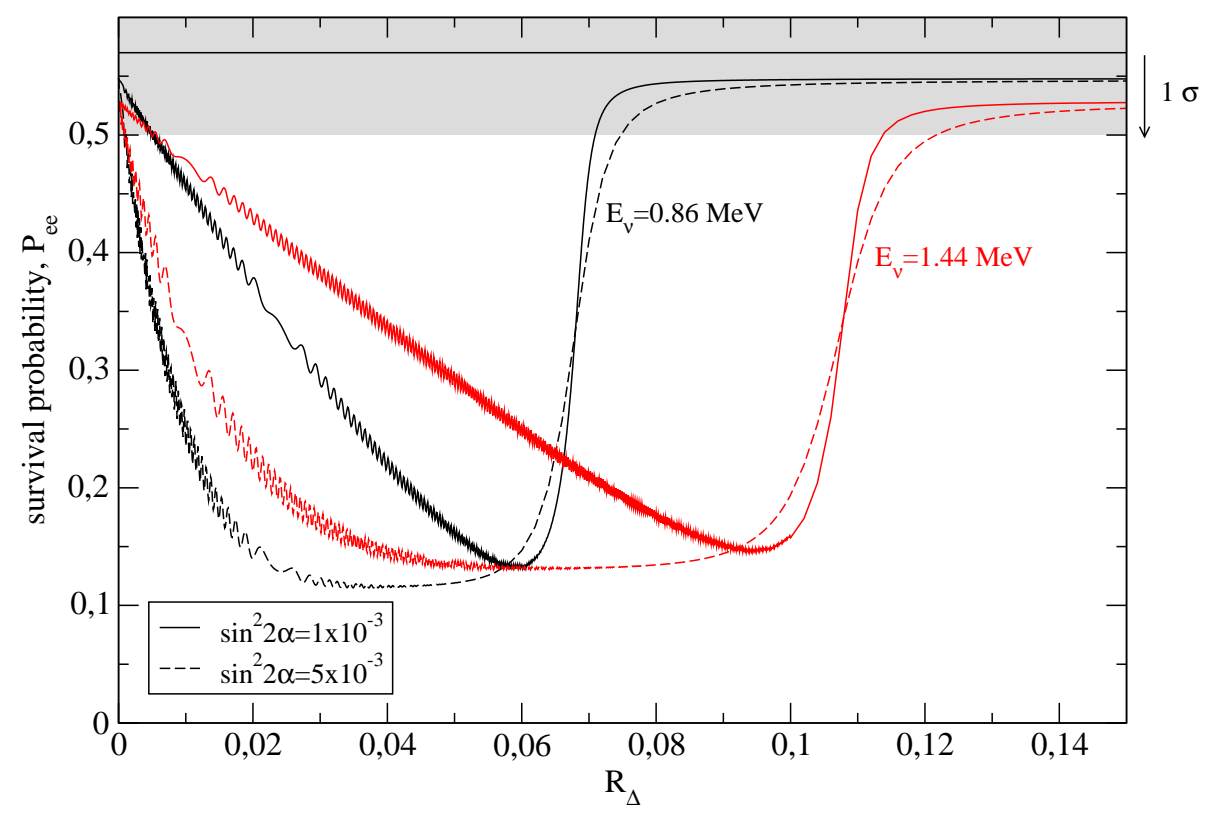

Figure 7: The survival probabilities for the monoenergetic ${ }^{7} B e-($ red $)$ and pep- (black) neutrino fluxes as functions of $R_{\Delta}$, for two values of mixing: $\sin ^{2} 2 \alpha=1 \times 10^{-3}$ (solid lines) and $5 \times 10^{-3}$ (dashed lines). The active neutrino oscillation parameters are the same as in fig. 3. The horizontal line and shadowed band show the central value and $1 \sigma$ band for the suppression factor determined by Borexino. 


\subsection{Upturn of the boron neutrino spectrum}

Using the survival probabilities obtained in sect. 2 we have computed the energy spectra of events for different experiments with and without sterile neutrino. These spectra together with experimental data are presented in figs. 8 - 12. We did not searched for the best fit of the data points, and the figures have an illustrative character. Notice that due to uncertainty in the original boron neutrino flux the experimental points can be shifted with respect to the theoretical lines by about $15 \%$.

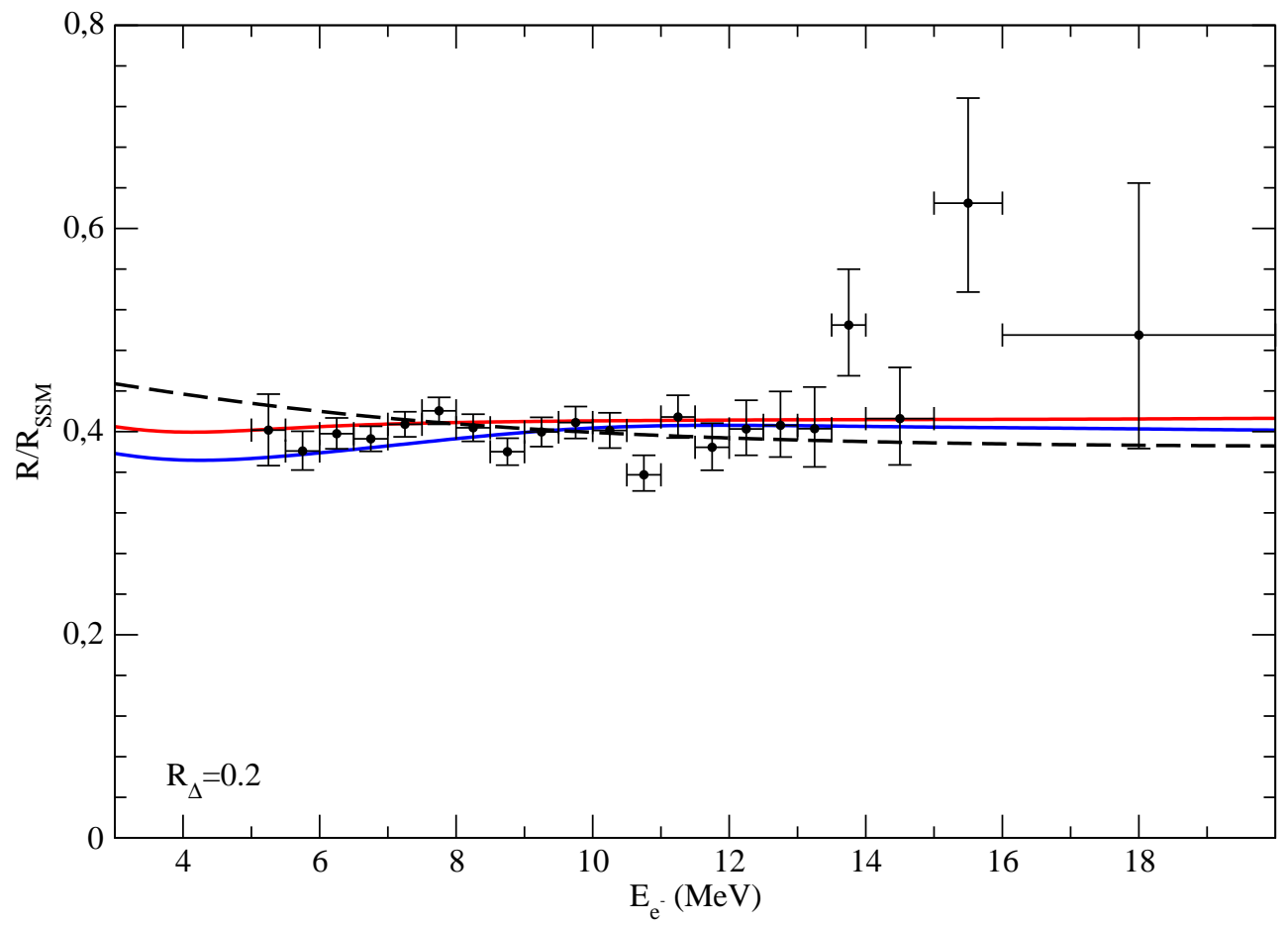

Figure 8: Prediction for the SuperKamiokande-I spectrum versus experimental data [6]. The active neutrino parameters are the same as before; the sterile neutrino parameters equal $R_{\Delta}=0.20$ and $\sin ^{2} 2 \alpha=1 \times 10^{-3}$ (red line) and $5 \times 10^{-3}$ (blue line). The pure LMA spectrum is presented by the dashed black line, with a normalization factor $f=0.91$ to reproduce the total observed number of events. We use the ${ }^{8} B$-neutrino flux according to the GS98 solar model [24].

In figs. 8 and 9 we show the ratio of the number of events in SuperKamiokande-I (SK-I) with and without oscillations for two different values of $R_{\Delta}$. Different curves correspond to the standard LMA solution (dashed) and the spectra with conversion to sterile neutrino. In 


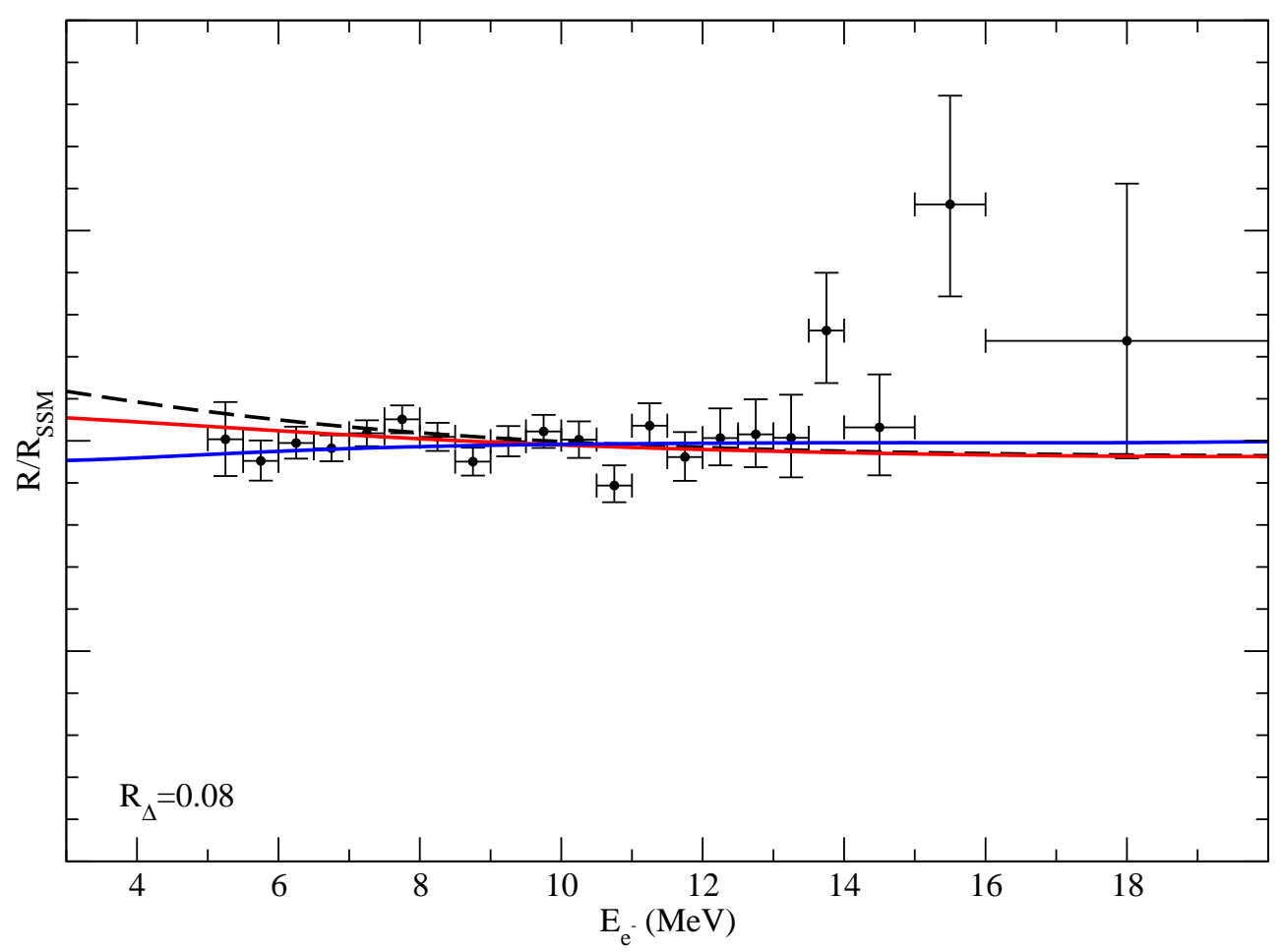

Figure 9: The same as in fig. 8 for $R_{\Delta}=0.08$.

the presence of sterile neutrino mixing the upturn can be completely eliminated and even transformed into turn down of the spectrum. In fig. 8 the dip at $E \sim 4 \mathrm{MeV}$ corresponds to the dip in the probability at approximately the same energy as in the fig. 3 (middle pannel). The difference of the predictions with and without sterile neutrino can be as big as $(15-20) \%$ at $E_{e}<5 \mathrm{MeV}$.

The SuperKamiokande-III data (SK-III) (fig. 10) has additional lower energy [14], however statistics is lower than in SK-I. Again there is no clear indication of the upturn in the SK-III spectrum and theoretical lines with sterile neutrino mixing can describe the data better than pure LMA solution.

SNO (fig. 11) is more sensitive to distortion of the neutrino spectrum. However, the dip in the electron spectrum is shifted to low energies by the threshold of the CC reaction on the deuteron: $E=1.44 \mathrm{MeV}$. Experimental points are from the SNO-LETA charge current event analysis [15]. Two low energy points of the spectrum show a sharp turn down. This can not be reproduced by the proposed dip, although with the dip the description is better 1. Also the Borexino spectrum (fig. 12) can be fitted better in presence of sterile neutrino mixing.

\footnotetext{
${ }^{1}$ Too sharp decrease of signal in the lowest energy bins is probably statistical fluctuations or some systematics.
} 


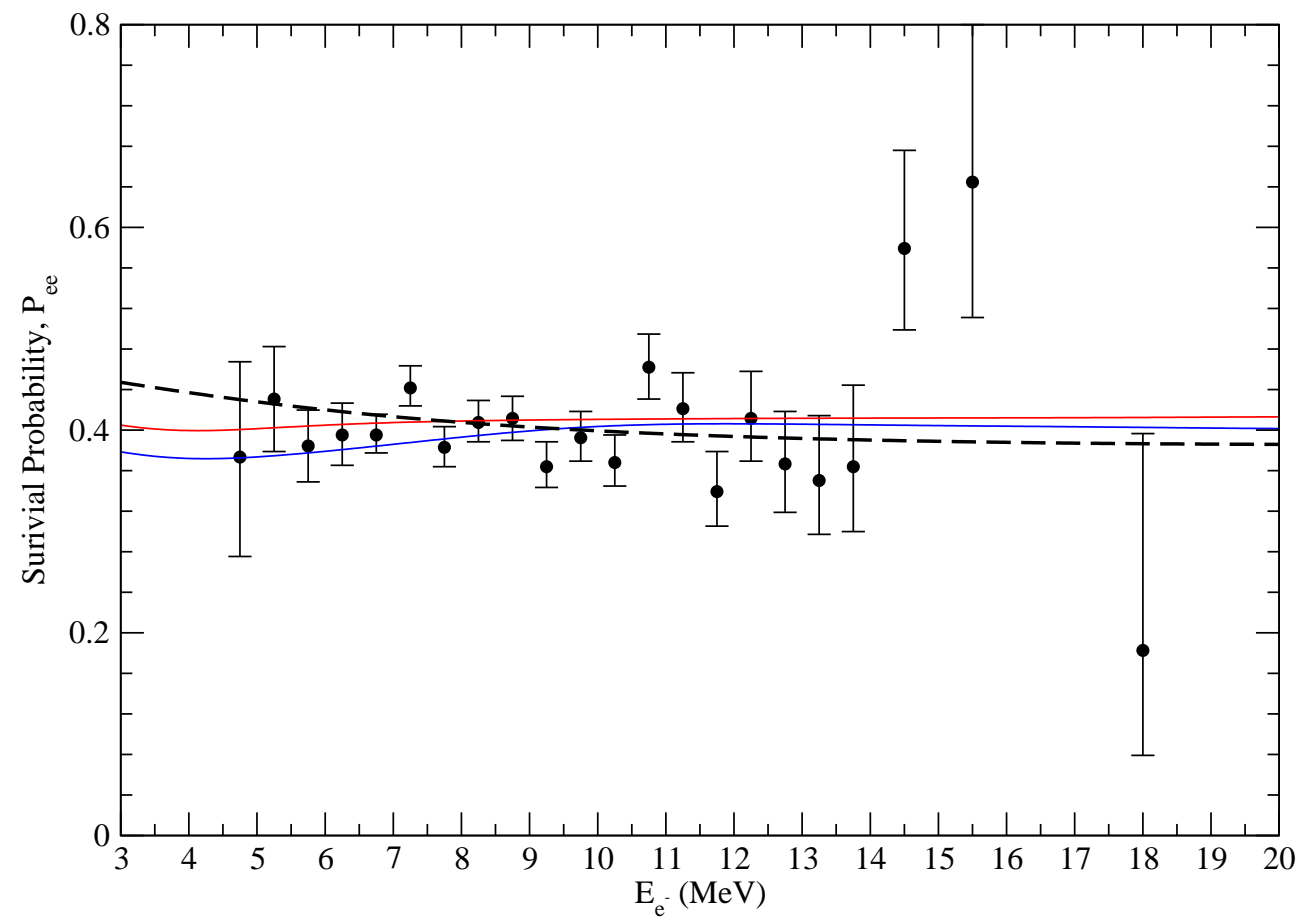

Figure 10: Prediction for SuperKamiokande-III spectrum versus experimental data [14. The neutrino parameters and the solar model as well as the normalization factor for pure LMA spectrum are the same as in fig. 8 (left).

According to fig. 8 - 12 an improved description of the data can be achieved with

$$
\Delta m_{01}^{2} \geq 1.5 \cdot 10^{-5} \mathrm{eV}^{2}, \quad \sin ^{2} 2 \alpha \sim 10^{-3} .
$$

\subsection{Further tests}

With higher statistics Borexino will improve precision of measurements of the boron neutrino spectrum. Also SuperKamiokande will achieve better measurements of spectrum at lower energies. The KamLAND solar [25] and $S N O+$ [26] experiments will further check the presence of the dip.

Additional probe of the existence of sterile neutrino (and restriction on its parameters) can be provided by measurements of the pep- neutrino line with $E=1.44 \mathrm{MeV}$ since the $p e p$-neutrino flux is known with high precision. In fig. 7 we show dependence of the suppression factor for the pep-neutrinos as function of $R_{\Delta}$. With increase of $R_{\Delta}$ the dip shifts to higher energies. In the interval $R_{\Delta}=0.07-0.11$, the $B e-$ neutrino flux has the LMA suppression, whereas the pep-flux can be suppressed by factor $0.15-0.20$ (the LMA suppression is 0.52 ). In the range $R_{\Delta}>0.12$ both fluxes have the LMA suppression. 


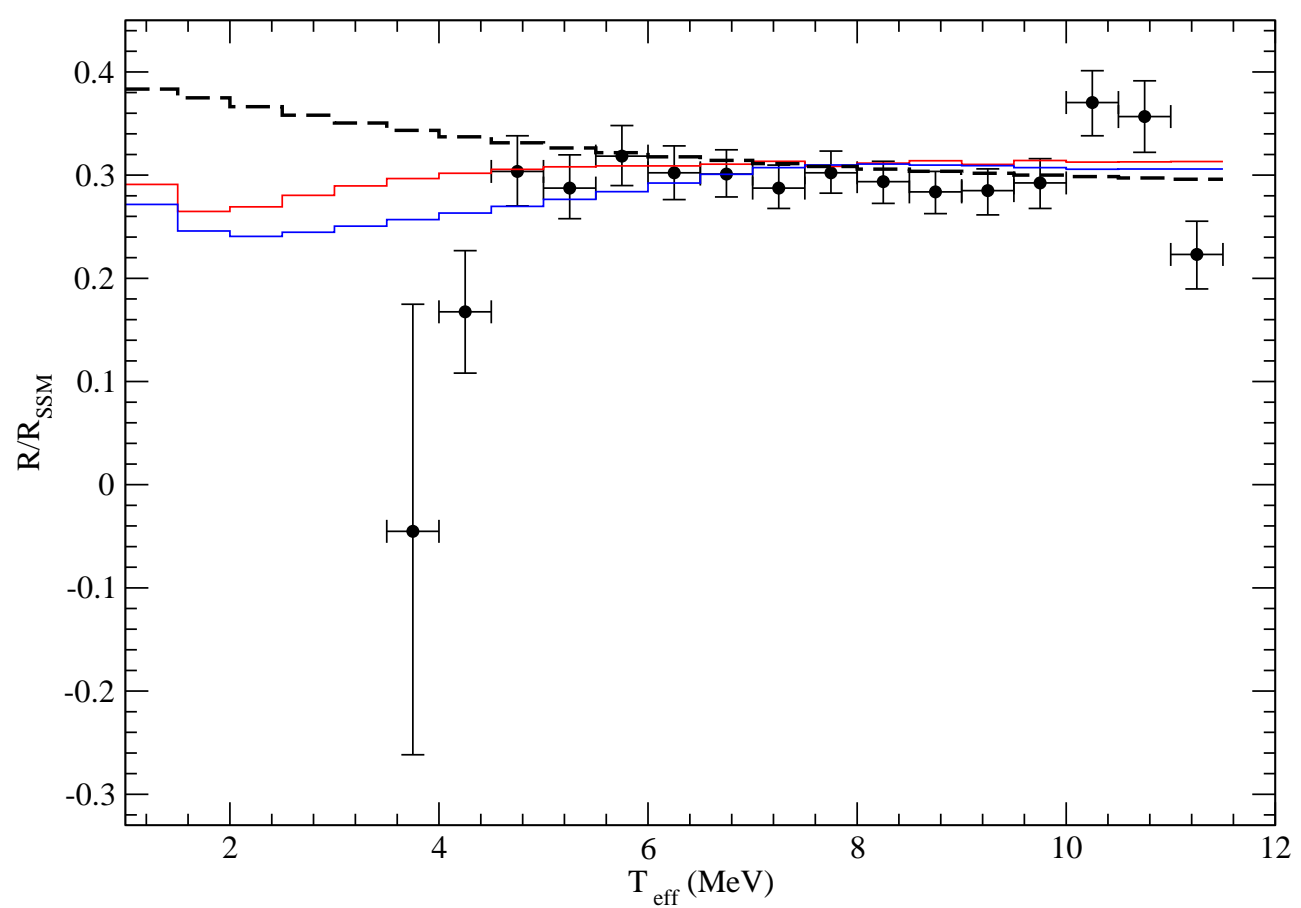

Figure 11: Prediction for the SNO-LETA electron spectrum versus experimental data [15]. The neutrino parameters and solar model are the same as in fig. 8 .

In the range $R_{\Delta}>0.12$ the $C N O$-neutrinos are sensitive to the presence of the dip, however the original fluxes of these neutrinos are not well known.

\section{Extra radiation in the Universe and $\nu_{s}-\nu_{3}$ mixing}

Smallness of mixing of the sterile neutrino in the states $\nu_{1}$ or/and $\nu_{2}\left(\left|U_{s i}\right|^{2}<10^{-3}\right)$ does not lead to production of significant concentration of $\nu_{s}$ in the Early Universe via neutrino oscillations [13. However, substantial abundance of $\nu_{s}$ can be generated if $\nu_{s}$ mixes in the state $\nu_{3}$ and $U_{s 3}$ is large enough. Description of the solar neutrino data presented in the previous sections does not change substantially, if $\nu_{s}$ mixes with combination

$$
\nu_{\tau}^{\prime} \equiv \cos \theta_{23} \nu_{\tau}+\sin \theta_{23} \nu_{\mu} \approx \nu_{3}
$$

where $\theta_{23}$ is the standard $2-3$ mixing angle. The $\nu_{s}-\nu_{\tau}^{\prime}$ mixing can be parametrized by the angle $\beta$ as

$$
\nu_{3} \approx \cos \beta \nu_{\tau}^{\prime}+\sin \beta \nu_{s}, \quad \nu_{0} \approx \cos \beta \nu_{s}-\sin \beta \nu_{\tau}^{\prime},
$$

so that $U_{s 3} \approx \sin \beta$. Here we neglect small rotations by the angles $\alpha$ and $\theta_{13}$ which do not influence conclusions of this section. (These mixings can be introduced before or after the 


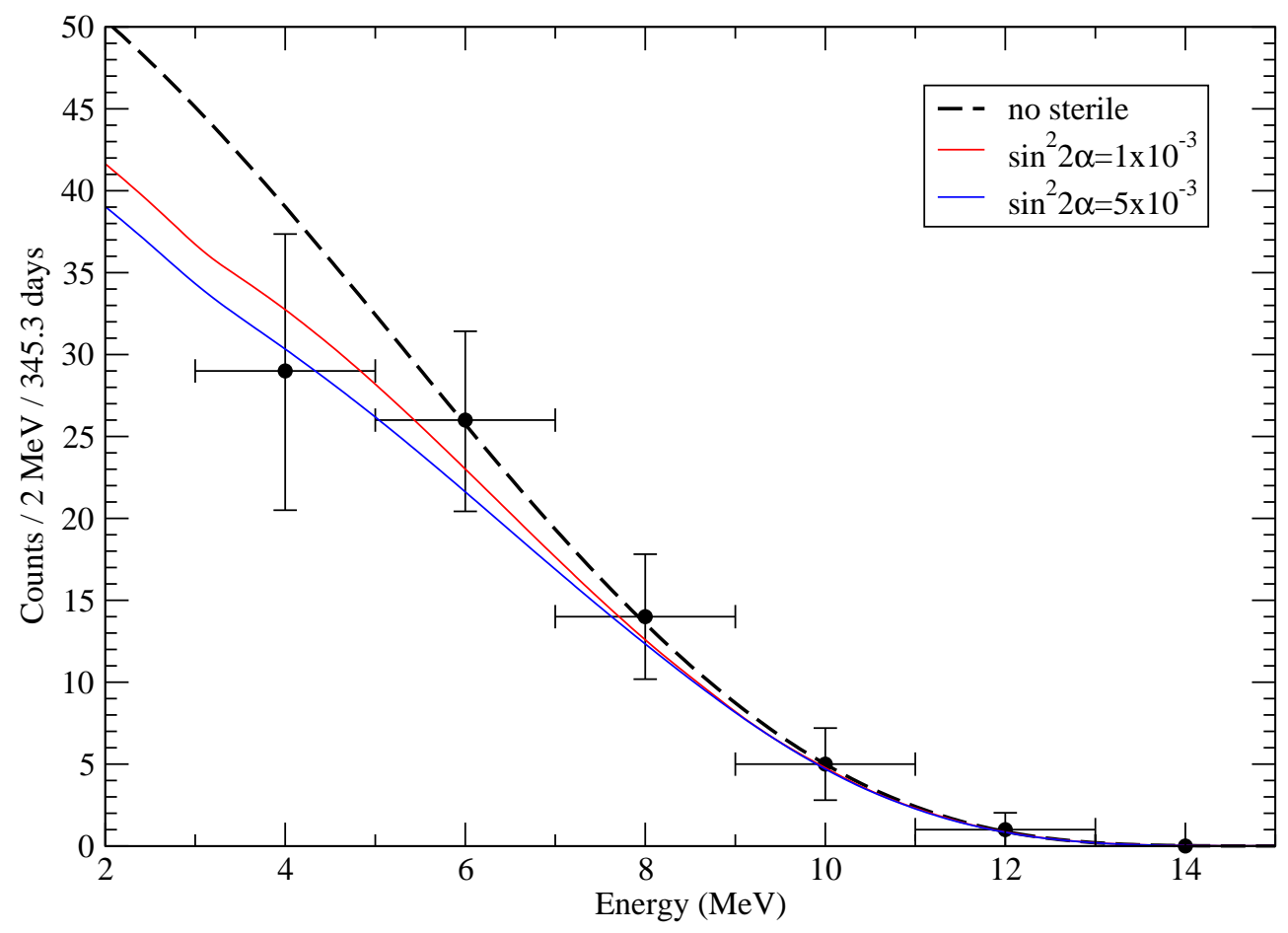

Figure 12: Prediction for $B$-neutrino spectrum at Borexino versus with experimental data [16]. The neutrino parameters and solar model are the same as in fig. 8 .

rotation ( $(\underline{36}))$. Since $\Delta m_{01}^{2} \ll \Delta m_{21}^{2} \ll \Delta m_{31}^{2}$, the mass squared difference of $\nu_{3}$ and $\nu_{0}$ equals

$$
\Delta m_{30}^{2} \approx \Delta m_{31}^{2}=2.5 \cdot 10^{-3} \mathrm{eV}^{2} .
$$

For this value of $\Delta m_{30}^{2}$ the mixing angle $\beta$ is restricted by the atmospheric neutrino data 27]:

$$
\sin ^{2} \beta \leq 0.2-0.3, \quad(90 \% \text { C.L. })
$$

and by the MINOS searches for depletion of the neutral current events [28]. For zero 1-3 mixing the bound $\beta<28.8^{\circ}$ has been established [28] which corresponds to

$$
\sin ^{2} \beta \leq 0.23, \quad(90 \% \text { C.L }) .
$$

In the presence of non-zero 1-3 mixing the bound becomes much weaker.

If $\sin ^{2} \beta \sim 0.2$, then according to [29] the sterile neutrinos practically equilibrate before the $\mathrm{BBN}$ epoch both in the resonance channel and in non-resonance channels, i.e. in neutrino and antineutrino channels. Consequently, in the epoch of nucleosynthesis and latter the additional effective number of neutrinos is

$$
\Delta N_{e f f} \approx 1
$$


The value $\Delta N_{\text {eff }} \approx 0.8$ can be obtained for $\sin ^{2} \beta \approx 0.03$ in the non-resonance channel and $\sin ^{2} \beta \sim 10^{-3}$ in the resonance channel. According to [27] $\Delta N_{\text {eff }} \approx 0.8$ is generated, if the $\nu_{s}-\nu_{\mu}$ mixing is about $\sin ^{2} \beta=0.02$.

The CNGS experiment has also some potential to restrict $\sin ^{2} \beta$ [30].

Let us consider other phenomenological consequences of the $\nu_{\tau}^{\prime}-\nu_{s}$ mixing. The level crossing scheme can be obtained from fig. 1 by adding the third active neutrino level and expanding whole the picture to the left. With increase of density the $\lambda_{2}$ increase until the the region of 1-3 resonance and then turns down and decreases in parallel to $\lambda_{1}$. Consequently, the sterile level $\lambda_{s} \approx \lambda_{0}$ (horizontal line) will cross $\lambda_{2}$ at some density above the 1-3 resonance density. Thus the mixing of $\nu_{s}$ in $\nu_{3}$ leads to appearance of the resonance in $\nu_{\tau}^{\prime}-\nu_{s}$ channel (normal mass hierarchy) at the density determined by

$$
V_{n}=\frac{1}{\sqrt{2}} G_{F} n_{n} \approx \frac{\Delta m_{03}^{2}}{2 E} \approx \frac{\Delta m_{31}^{2}}{2 E} .
$$

In the isotopically neutral medium this density is about 2 time larger than the density of 1-3 resonance. For the inverted mass hierarchy the resonance appears in the antineutrino channel $\bar{\nu}_{\tau}^{\prime}-\bar{\nu}_{s}$.

Inside the Earth the $\nu_{\tau}^{\prime}-\nu_{s}$ resonance energy equals $E \approx 12 \mathrm{GeV}$ and wide resonance peak appears in the range $(10-15) \mathrm{GeV}$. This can be tested in studies of the atmospheric neutrinos (spectra, zenith angle dependences) in the IceCube DeepCore detector [31] and in next generation Megaton-scale experiments [32. Effect of such a mixing should show up in the long baseline experiments as the energy dependent disappearance of the $\nu_{\mu}$-flux.

The $\nu_{\tau}^{\prime}-\nu_{s}$ mixing also influences the supernova (SN) neutrino conversion. The corresponding level crossing in the collapsing star will be adiabatic (at least before shock wave arrival) and therefore $\nu_{\tau}^{\prime}$ converts almost completely in this resonance into $\nu_{s}$. At larger distances from center of a star this $\nu_{s}$-flux will encounter the lower density $\nu_{s}$ resonances due to the crossing of $\nu_{s}$ and $\nu_{1 m}$ levels (see fig. 1). The latter will lead to partial conversion of $\nu_{s}$ into $\nu_{e}$, since the adiabaticity is broken in these resonances. Hence, the following chain of transitions is realized:

$$
\nu_{\tau}^{\prime}\left(\nu_{\mu}, \nu_{\tau}\right) \rightarrow \nu_{s} \rightarrow \nu_{s}, \nu_{e}
$$

Consequently, even for relatively large 1-3 mixing which leads to the transition $\nu_{e} \rightarrow \nu_{3}$ with $\left|\left\langle\nu_{e} \mid \nu_{3}\right\rangle\right|^{2} \ll 1$ (for normal mass hierarchy), the $\nu_{e}$ signal may not be strongly suppressed due to conversion described in (37). In the case of inverted mass hierarchy similar consideration holds for the antineutrino channels.

In this consideration for simplicity we have neglected possible collective effects due to neutrino-neutrino scattering and effects of shock wave propagation (see [32]). 


\section{Conclusions}

1. Recent measurements of the energy spectra of the solar neutrino events at SuperKamiokande, SNO, Borexino do not shown the expected (according to LMA) upturns at low energies. The absence of the upturn can be explained by mixing of very light sterile neutrino in the mass states $\nu_{1}$ or $/$ and $\nu_{2}$ with $\Delta m_{01}^{2} \sim(0.7-2) \cdot 10^{-5} \mathrm{eV}^{2}\left(R_{\Delta}=0.07-0.25\right)$ and mixing $\sin ^{2} 2 \alpha=(1-5) \cdot 10^{-3}$. Such a mixing leads to appearance of the dip in the $\nu_{e}-$ survival probability in the energy range $(1-7) \mathrm{MeV}$, thus removing the upturn of the spectra. For $\Delta m_{01}^{2} \sim 2 \cdot 10^{-5} \mathrm{eV}^{2}$ and $\sin ^{2} 2 \alpha \sim 5 \cdot 10^{-3}$ the $\nu_{e}-\nu_{s}$ conversion can even produce a turn down of the spectra. Description of the existing solar neutrino data in the presence of mixing with sterile neutrino is apparently improved.

Values of $\Delta m^{2}<0.6 \cdot 10^{-5} \mathrm{eV}^{2}$ (for mixing angle interval $\sin ^{2} 2 \alpha=(1-5) \cdot 10^{-3}$ ) are excluded by the Borexino measurements of the $B e-$ neutrino flux. The presence of the dip can be further tested in future precision measurements of the low energy part of the $B$-neutrino spectrum as well as the pep- neutrino flux.

2. Mixing of $\nu_{s}$ in the $\nu_{3}$ mass eigenstate with $\left|U_{s 3}\right|^{2} \sim 0.02-0.2$ leads to production of significant concentration of $\nu_{s}$ via oscillations in the Early Universe. For $\left|U_{s 3}\right|^{2} \sim 0.1-0.2$ nearly equilibrium concentration can be obtained both in neutrino and antineutrino channels thus generating additional effective number of neutrinos $\Delta N_{\text {eff }} \sim 1$ before the BBN epoch. This can explain recent cosmological observations.

3. Mixing of $\nu_{s}$ in $\nu_{3}$ leads to a number of phenomenological consequences, in particular, it can affect the atmospheric and accelerators neutrino fluxes as well as fluxes of the SN neutrinos. The mixing leads to existence of the $\nu_{s}-\nu_{\tau}^{\prime}$ resonance. For neutrinos crossing the Earth the resonance should appear at energies $E \sim 10-15 \mathrm{GeV}$. This can be tested in future atmospheric neutrino studies with Megaton-scale detectors as well in the long baseline experiments with accelerator neutrino beams.

\section{Acknowledgments}

P. C. de Holanda is grateful to the AS ICTP for hospitality during his visit where most part of this paper has been accomplished.

\section{Appendix A: Wiggles}

As we described in sect. 2, wiggles in the dependence of the $\nu_{e}$-survival probability on energy are the result of interference of the amplitudes which contribute to the same $\nu_{e} \rightarrow \nu_{1}$ 
transition. The zoomed view of the survival, $P_{e e}$, and transition, $P_{e s}$, probabilities is shown in fig. 13. The period of wiggles is about (0.5 - 0.6) MeV.

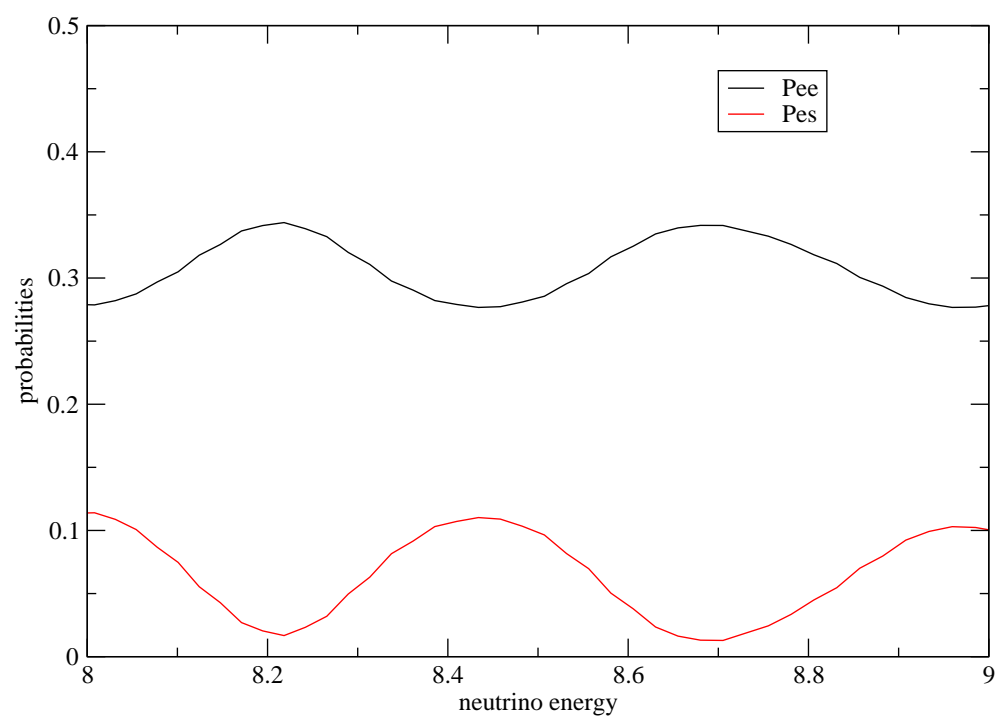

Figure 13: A zoomed view of the survival and transition probabilities in the energy range where the wiggles can be well seen. The sterile neutrino parameters equal $R_{\Delta}=0.25$ and $\sin ^{2} 2 \alpha=10^{-3}$.

The key feature which leads to the wiggles with rather large period in the energy scale and therefore prevents them from being averaged out at the integration over the production region is that the interference phase is collected over relatively small distances $L$. These are the distances between the production point and the low density $\nu_{s}$-resonance or the distance between the two $\nu_{s}$ resonances as can be seen in the fig. 14. For the neutrino energy $E \sim 8 \mathrm{MeV}$ the distance $L \approx 20 l_{m}$ where $l_{m}$ is the oscillation length in matter. Therefore the period of wiggles can be estimated as $\Delta E / E \sim l_{m} / L \sim 1 / 20$ in agreement with results of fig. 14.

The wiggles are partially averaged due to integration over the production region. Notice that with decrease of $\Delta m_{01}^{2}$ the lower resonance shifts to lower densities and the distance $L$ increases leading to smaller period of wiggles and stronger averaging. This is one of the reasons of disappearance of wiggles with decrease of $\Delta m_{01}^{2}$. The amplitude of wiggles also decreases with increase of $\alpha$ : the latter means better adiabaticity and therefore suppression of the contribution of one of the channels responsible for interference. 


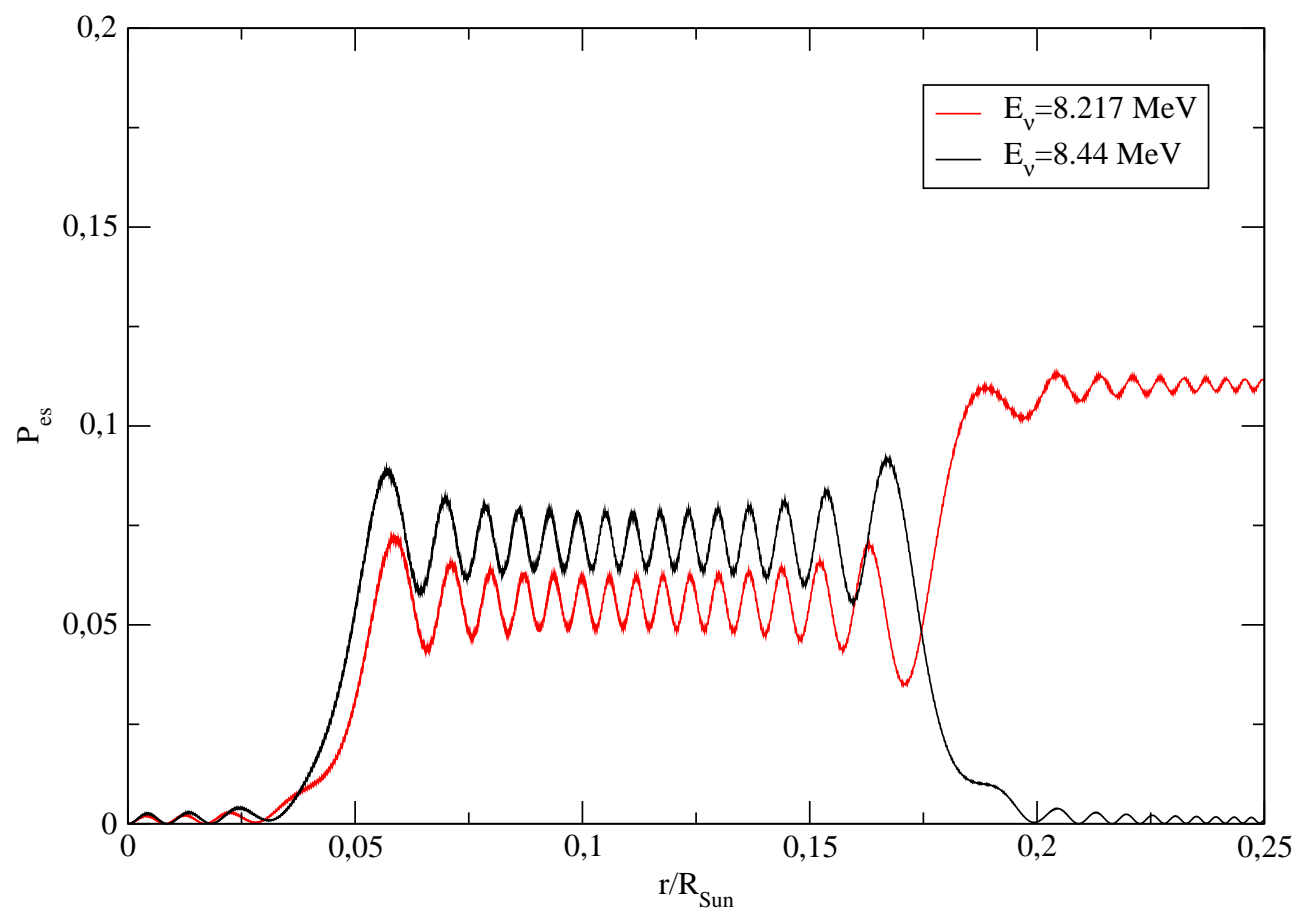

Figure 14: Transition probability to sterile neutrino for neutrino created at the Sun center as function of distance from the center of the Sun. The sterile neutrino parameters are $R_{\Delta}=0.25$ and $\sin ^{2} 2 \alpha=10^{-3}$.

\section{References}

[1] L. Wolfenstein, Phys. Rev. D17 (1978) 2369; in "Neutrino -78", Purdue Univ. C3, 1978 .

[2] S. P. Mikheyev and A. Yu. Smirnov, Sov. J. Nucl. Phys. 42 (1985) 913; Nuovo Cim. C9 17, (1986); Zh. Eksp. Teor. Fiz. 91, 7 (1986), [Sov. Phys. JETP 64, 4 (1986)] reprinted in [arXiv:0706.0454 [hep-ph]].

[3] B. T. Cleveland, et al., Astrophys.J. 496, 505 (1998).

[4] J. N. Abdurashitov et al. [SAGE Collaboration], Phys. Rev. C 80 (2009) 015807 arXiv:0901.2200 [nucl-ex]].

[5] M. Altmann et al. [GNO Collaboration], Phys. Lett. B 616 (2005) 174 arXiv:hep-ex/0504037].

[6] J. Hosaka et al. [Super-Kamkiokande Collaboration], Phys. Rev. D 73 (2006) 112001 arXiv:hep-ex/0508053. 
[7] B. Aharmim et al. [SNO Collaboration], Phys. Rev. C 72 (2005) 055502 arXiv:nucl-ex/0502021.

[8] B. Aharmim et al. [SNO Collaboration], Phys. Rev. C 75 (2007) 045502 arXiv:nucl-ex/0610020.

[9] B. Aharmim et al. [SNO Collaboration], Phys. Rev. Lett. 101 (2008) 111301 arXiv:0806.0989 [nucl-ex]].

[10] C. Arpesella et al. [The Borexino Collaboration], Phys. Rev. Lett. 101 (2008) 091302 arXiv:0805.3843 [astro-ph]].

[11] S. Abe et al. [KamLAND Collaboration], Phys. Rev. Lett. 100 (2008) 221803 arXiv:0801.4589 [hep-ex]].

[12] A. Gando et al., arXiv:1009.4771 [hep-ex].

[13] P. C. de Holanda and A. Y. Smirnov, Phys. Rev. D 69 (2004) 113002 arXiv:hep-ph/0307266.

[14] K. Abe et al., arXiv:1010.0118 [hep-ex].

[15] B. Aharmim et al. [SNO Collaboration], Phys. Rev. C 81 (2010) 055504 arXiv:0910.2984 [nucl-ex]].

[16] G. Bellini et al. [The Borexino Collaboration], Phys. Rev. D 82 (2010) 033006 arXiv:0808.2868 [astro-ph] v.3].

[17] E. Komatsu et al., arXiv:1001.4538 [astro-ph.CO].

[18] J. Dunkley et al., arXiv:1009.0866 [astro-ph.CO].

[19] J. Hamann, S. Hannestad, G. G. Raffelt, I. Tamborra and Y. Y. Y. Wong, Phys. Rev. Lett. 105 (2010) 181301 [arXiv:1006.5276 [hep-ph]].

[20] U. Seljak, A. Slosar and P. McDonald, JCAP $0610 \quad$ (2006) 014 arXiv:astro-ph/0604335].

[21] Y. I. Izotov and T. X. Thuan, Astrophys. J. 710 (2010) L67 arXiv:1001.4440 [astroph.CO]].

[22] G. Steigman, JCAP 1004 (2010) 029 [arXiv:1002.3604 [astro-ph.CO]].

E. Aver, K. A. Olive and E. D. Skillman, JCAP 1005 (2010) 003 arXiv:1001.5218 [astro-ph.CO]]; arXiv:1012.2385 [astro-ph.CO].

[23] L. M. Krauss, C. Lunardini and C. Smith, arXiv:1009.4666 [hep-ph].

K. Nakayama, F. Takahashi and T. T. Yanagida, arXiv:1010.5693 [hep-ph]. 
[24] J. N. Bahcall, S. Basu, M. Pinsonneault and A. Serenelli Astrophys. J. 618 (2005) 1049; N. Grevesse and A. J. Sauval, Space Sci. Rev. 85 (1998) 161.

[25] Y. Kishimoto [KamLAND Collaboration], J. Phys. Conf. Ser. 120 (2008) 052010.

[26] M. C. Chen [SNO+ Collaboration], arXiv:0810.3694 [hep-ex]. C. Kraus and S. J. M. Peeters [SNO+ Collaboration], Prog. Part. Nucl. Phys. 64 (2010) 273 .

[27] M. Cirelli, G. Marandella, A. Strumia and F. Vissani, Nucl. Phys. B 708 (2005) 215 arXiv:hep-ph/0403158.

[28] P. Adamson et al. [The MINOS Collaboration], Phys. Rev. D 81 (2010) 052004 arXiv:1001.0336 [hep-ex]].

[29] A. D. Dolgov and F. L. Villante, Nucl. Phys. B 679 (2004) 261 arXiv:hep-ph/0308083.

[30] A. Donini, M. Maltoni, D. Meloni, P. Migliozzi and F. Terranova, JHEP 0712 (2007) 013 arXiv:0704.0388 [hep-ph]].

[31] O. Schulz [IceCube Collaboration], AIP Conf. Proc. 1085 (2009) 783.

C. Wiebusch and f. t. I. Collaboration, arXiv:0907.2263 [astro-ph.IM].

[32] P. C. de Holanda and A. Yu. Smirnov, in preparation. 